\title{
Flow-induced transverse vibration of a circular cylinder close to a plane wall at small gap ratios
}

\author{
L.F. Chen ${ }^{\mathrm{a}}$, G.X. Wu ${ }^{\mathrm{b}, *}$ \\ ${ }^{a}$ School of Naval Architecture and Ocean Engineering, Jiangsu University of Science and Technology, China \\ ${ }^{b}$ Department of Mechanical Engineering, University College London, Torrington Place, London WC1E7JE, UK
}

\begin{abstract}
Numerical simulations of flow-induced transverse vibration of a near-wall cylinder with small gap to diameter ratios $(e / D \leq 0.5)$ at $R e=200$ are performed based on the Navier-Stokes equations using a finite volume method based OpenFOAM codes. Multi-block mesh is used. As the cylinder is very close the wall, remeshing is regularly applied during the body oscillation to avoid over distortion of the grid. A model to account for the collision of the cylinder with the wall is adopted, in which when the gap between the cylinder and the wall is smaller than a critical value, the direction of its velocity is reversed. Simulations are made for low mass ratio, relevant to the cases in hydrodynamics. The results show that the motion amplitude generally increases with the reduced velocity $U^{*}=U /\left(f_{n} D\right)$ first and then decreases after reaching a peak, and there is no obvious hysteretic transition. When $e / D$ decreases, the largest amplitude decreases, and the reduced velocity at which the largest amplitude occurs increases. The results also indicate that the wall has a significant effect on the natural frequency of the cylinder, suggesting that the fluid force has components strongly affected by the body acceleration and displacement. When $e / D$ decreases, the frequency at which the largest amplitude occurs becomes larger than the natural frequency obtained from the ratio of the cylinder stiffness to the summation of mass and the added mass. It is observed that, in all the cases when $e / D \leq 0.5$, the anti-clockwise vortices in the wake of the cylinder are suppressed and only one single vortex of $1 \mathrm{~S}$ type is shed downstream. As $e / D$ decreases, the clockwise vortex shed from the upper part of the cylinder remains strong, and the anti-clockwise vortex from the lower part of the cylinder gradually becomes weaker, and the vortex street is less deflected upwards.
\end{abstract}

Keywords: Flow-induced vibration, small gap ratio, cylinder collision with the wall, amplitude branch, vortex shedding

\section{Introduction}

Owing to uneven nature of seafloor topography or randomness of scouring, subsea pipelines are often laid across escarpment, trough or depressions over the seafloor. These free span pipelines would undergo flowinduced vibration (FIV) due to a current or other kinds of flows near the seafloor. Even though the vibration 5 amplitude may not be always large, the persistent vibration, especially the high frequency components, over a long period of time, can lead to severe fatigue damage (Tsahalis (1983) [1]; Sumer and Fredsoe (2006) 2]). Therefore, the FIV of the free span pipeline has been a major consideration in the design of subsea pipeline system. From the hydrodynamic point of view, a cylinder close to the seafloor means that its FIV is very much affected by shape of the seafloor and the boundary shear flow, and it may regularly collide with the seafloor and bounce back.

For a fixed circular cylinder close to a plane wall, the behaviour of flow past the cylinder and the force on the cylinder have been investigated through a substantial volume of work. Bearman and Zdravkovich

\footnotetext{
* Corresponding author.

Email address: g.wu@ucl.ac.uk (G.X. Wu)
} 
(1978) 3] carried out experimental studies on the influence of the gap, $e$, between the bottom of the cylinder of diameter $D$ and the seabed on the vortex shedding for Reynolds number $R e$ from $2.5 \times 10^{4}$ to $4.8 \times 10^{4}$, 15 and found that the vortex shedding in the wake of the circular cylinder was suppressed by the gap when $e / D<0.3$. Zdravkovich (1985) 4 and Lei et al. (1999) [5] later measured the drag and lift forces on the circular cylinder close to a plane wall for $4.8 \times 10^{4}<R e<3 \times 10^{5}$ and $1.31 \times 10^{4}<R e<1.4 \times 10^{4}$ respectively. They both found that both the drag and lift force coefficients, $C_{D}$ and $C_{L}$, strongly depended on the gap ratio $e / D$ when the main part of the cylinder was in the shear flow and the drag was almost 20 unaffected by the gap ratio when only the bottom of the cylinder was in or near the shear flow. From the magnitude of the root-mean-square (RMS) lift coefficient $C_{L, r m s}$ Lei et al. (1999) also observed the onset or suppression of the vortex shedding. Wang and Tan (2008) [6] investigated the wake flow characteristics of a cylinder close to a flat plane with $0.1 \leq e / D<1.0$ for $R e=1.2 \times 10^{4}$ and $\delta / D=0.4$, where $\delta$ is boundary layer thickness of the wall at the location of the cylinder. Their results showed that both the 25 ensemble-averaged (including the mean velocity vectors and Reynolds stress) and the instantaneous flow fields were strongly dependent on $e / D$, and that the wake flow and the nature of flow asymmetry about the cylinder centerline will change with the gap ratio for $e / D<=0.6$. In addition to experimental study, numerical simulations of the flow past a cylinder close to a flat plane have also been extensively undertaken. Lei et al. (1999) [5] did computation for Reynolds number Re < 1000. Zhao et al. (2007) [7, Ong et al. 30 (2012) 8 performed the simulation for $R e \sim O\left(10^{4}\right)$. Their results showed a good qualitative agreement with the published experimental data. Ong et al. (2010) [9] carried out a numerical study for $\operatorname{Re}=3.6 \times 10^{6}$ and found that the vortex shedding was suppressed as well at this Reynolds number when the gap was smaller than a critical one, and this critical gap was smaller at the larger Re. Prsic et al. (2016) 10 performed simulations using large eddy simulations (LES) for the flow around a circular cylinder close to a flat seabed 35 at $R e=1.31 \times 10^{4}$. They found that for an intermediate $e / D=0.6$, the larger $\delta / D$, equivalent to cylinder at different location, led to lower mean $C_{D}$ and $C_{L, r m s}$, as well as more elongated wake vortices.

The FIV of a free span pipeline, which includes vortex induced vibration (VIV), is usually modeled as a spring-mounted cylinder near a plane wall. Tsahalis and Jones 11] performed model tests on the FIV of a flexible pipe, and found that, in the presence of a plane wall, the maximum amplitude of vibration was

40 reduced. Fredsoe et al. $[12$ carried out a series of experiments with gap ratio $0<e / D<1$. It was found that the frequency of the vibration was close to vortex shedding frequency $f_{v}$ of a fixed cylinder when the reduced velocity $U^{*}=U /\left(f_{n} D\right)$ (where $U$ was the velocity of incoming flow and $f_{n}$ the natural frequency of the cylinder) was small $\left(U^{*}<3\right)$. When $3<U^{*}<8$ the frequency of the vibration was found to be considerably larger than $f_{v}$. They suggested that the vibration of the cylinder was partly caused by vortex 45 shedding, and partly by the force induced by the wall. Yang et al. 13 performed an experimental study on flow-induced vibrations of a cylinder near a plane boundary for $3600<R e<22500$. They observed that, with the gap increasing in the range of $0.06 \leq e / D \leq 4.69$, the vibration amplitude increases, while the frequency varied only slightly when $e / D>0.66$. They also found that there was a clear difference in amplitude and frequency responses of the cylinder between the larger $(e / D>0.66)$ and smaller $(e / D<0.3)$

50 gap ratios. Zhao and Cheng (2011) 14 performed a study on FIV of a near-wall cylinder with $e / D=0.002$ and 0.3 for $1=<U^{*} \leq 15$ and $1000 \leq R e \leq 15000$. They introduced a bounce-back model in which when the cylinder moved towards the wall and $e / D$ became very small, the velocity direction was reversed and its magnitude was changed through a bounce back coefficient. Based on the numerical results, three vortex shedding modes were identified depending on the reduced velocity. When $2.5 \leq U^{*} \leq 6$, the vortex shedding 55 was in a single-vortex mode, and the vortex was shed only from the upper part of the cylinder. When $9<U^{*} \leq 11$, in which the FIV amplitude increased with $U^{*}$, vortex-shedding-after-bounce-back mode occurred, and the vortices were shed from the lower part of the cylinder after the cylinder was bounced back from the plane wall. Vortex-shedding-before-bounce-back mode occurred in the range of the reduced velocity when resonance occurred. Wang et al. [15] conducted an experimental study on the free motion of a 60 near-wall cylinder with $0.05 \leq e / D \leq 2.5,3000 \leq R e \leq 13000$ and $1.53 \leq U^{*} \leq 6.62$. They found that the elastically mounted near-wall cylinder generally exhibited periodic motion and vortex shedding. However, the motion and vortex shedding showed non periodic features at some range of $U^{*}$, which indicated some direct effect of the wall. The vortices shed from the vibrating cylinder were arranged into a single-sided pattern. A numerical study on the wall effects at a low Reynolds number $(R e=100)$ for $0.5 \leq e / D \leq 10$ 
was presented by Tham et al. [16. Initial and lower branches in the amplitude curve against $U^{*}$ were found for $0.75 \leq e / D \leq 10$. At a low gap ratio of $e / D=0.5$, they observed two transverse amplitude peaks in the vibration response curve. They also found that the lock-in region became larger for lower e/D. Li et al. 17 . investigated the hydrodynamic forces, vibration characteristics, phase relations, response frequencies as well as vortex shedding patterns for a near-wall cylinder at $R e=200$ and $e / D=0.9$. They found that the effect of the wall increased the mean lift force and that the vortex shed from the lower part of the cylinder was suppressed by both the boundary layer of the wall and the vortex shed from the upper part of the cylinder.

The discussion above indicates that there has been a substantial volume of work on near-wall fixed and vibrating circular cylinders. Some of these have demonstrated that, unlike the cases of a fixed cylinder where the vortex shedding is suppressed below a critical gap ratio $(e / D \approx 0.3)$, the elastically mounted cylinder always vibrates and sheds vortex even at a very small gap ratio. For instance, the flow-induced vibration occurred at a subcritical Reynolds number even when the initial gap ratio was as small as $e / D=0.002$ [14 or $e / D=0.05[15$ at which the vortex shedding from a fixed cylinder was weak. For the near-wall cylinder at low Reynolds numbers, it was revealed that the effect of the wall changed the overall shape of the motions response curve against $U^{*}$ and that decrease of $e / D$ enlarged the lock-in region at $e / D \geq 0.5$. It is noted so here that far fewer studies on the motion response of a near-wall cylinder have been done for smaller $e / D$ $(e / D<0.5)$ at low Reynolds number, with which flow characteristics past the cylinder are different from those at $e / D \leq 0.5$. Chen and $\mathrm{Wu}(2019)[18$ has reported that the flow past a near-wall fixed circular cylinders at $R e=200$ is steady and no strong vortex shedding occurs when $e / D<0.5$. The present study undertakes in depth studies on flow-induced vibration of a free circular cylinder at the similar gap ratios

85 and the same Reynolds number. The result from Prisc et al.[10] indicated that the three-dimensionality had insignificant influence on the hydrodynamic forces of the near-wall cylinder when $R e<=500$, thus $2 D$ simulations are performed in the present study.

In particular, the vibrating cylinder near the wall may sometimes reach the wall and collide with the solid surface. When this occurs it is assumed that the cylinder will bounce back upon touching the surface. After 90 the cylinder is bounced back, the magnitude of its velocity does not change, and the direction of the velocity is reversed. In order to avoid mesh failure when the cylinder collides with the wall, a treatment used by Zhao and Cheng [14 is employed, in which the cylinder is forced to be bounced back if the distance between the cylinder bottom and the wall reaches a critical value. To have a quality mesh at a very small gap during the cylinder vibration, a remeshing technique is developed by means of open source OpenFOAM codes. It

95 makes coordinates of cell node to be rearranged according to the cylinder displacement at each time step and prevents from mesh violation occurrence at a very small gap. The technique enables simulations for a body very close to a wall to be successfully made.

\section{Mathematical model and numerical method}

In the present study, the governing equations for the flow and the body motion are simultaneously solved using a transient solver based on a Pressure Implicit with Splitting of Operators (PISO) algorithm and Newmark- $\beta$ method, by means of open source OpenFOAM codes. The methodology is summarized below.

\subsection{Governing equations and numerical method for fluid flow}

The two dimensional (2D) continuity equation and Navier-Stokes equations for the incompressible viscous flows are used. The non-dimensional forms of these equations, based on the incoming velocity $U$ of the flow, body dimension $D$ and density $\rho$ of the fluid, can be written as

$$
\begin{aligned}
\nabla \cdot \boldsymbol{u} & =0, \\
\frac{\partial \boldsymbol{u}}{\partial t}+(\boldsymbol{u} \cdot \nabla) \boldsymbol{u}+\nabla p-\frac{1}{R e} \nabla^{2} \boldsymbol{u} & =0,
\end{aligned}
$$

where $\boldsymbol{u}$ represents the velocity, $p$ denotes the pressure, $R e=U D / \nu$ is Reynolds number and $\nu$ is the

kinematic viscosity of the fluid. 


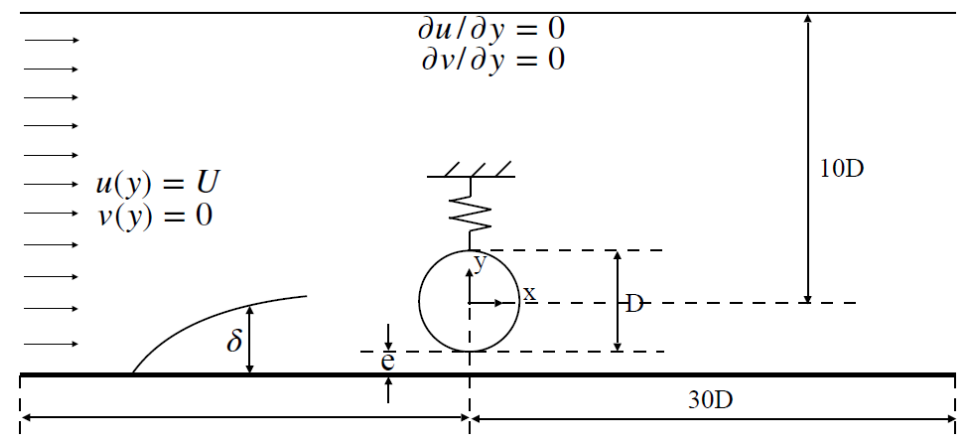

Figure 1: Skematic diagram of flow past a transversely vibrating circular cylinder very close to a plane wall.

A finite volume method is used for the spatial discretisation of continuity and Navier-Stokes equations and a Pressure Implicit with Splitting of Operators (PISO) algorithm[19] is used to deal with the coupling between Eqs.(1) and (2). The integration over each cell is applied to the governing equations, and the convection term and the diffusion term within each cell are converted to the integrations along the cell faces based on Gaussian theorem. All the variables are defined at the centre of each cell. The values required at each cell face are obtained through interpolation from the centres of the two cells that share this face 20. Here, Gaussian integration with linear upwind differencing interpolation scheme is applied to the convection term, and Gaussian integration with linear interpolation scheme and a limited non-orthogonal correction (correction coefficient 0.5) is adopted for the diffusion term. A least squares method with a cellLimited gradient limiter is applied to computing the pressure gradient. During the iterative procedures of the PISO algorithm, a preconditioned bi-conjugate gradient method with a diagonal incomplete-LU (asymmetric) preconditioner is used to solve the linear solver for $\boldsymbol{u}$. A generalized geometric-algebraic multi-grid method with a Gauss-Seidel smoother is used to solve the linear solver for the pressure $p$. For the time derivative $(\partial u / \partial t)$, a first-order implicit Euler scheme is chosen for the temporal advancement.

\subsection{Dynamic equations of motion}

In the present study, the cylinder of mass $m$ is assumed to be rigid, streamwise-fixed and transversely supported by a linear spring with constant structural damping $c$ and stiffness $k$ (see Fig. 11). The onedegree-of-freedom equation of the motion is given by

$$
m \ddot{Y}+c \dot{Y}+k Y=F_{L},
$$

where $Y$ is the cylinder displacement relative to the initial position in the direction perpendicular to that of the main incoming flow, $\ddot{Y}$ and $\dot{Y}$ respectively represent the second and first derivative of $Y$ with respect to $t, F_{L}$ denotes the transverse hydrodynamic force. Eq. (3) is numerically solved using a Newmark- $\beta$ method for constant time step. When $n=1$,

$$
\begin{aligned}
& \dot{Y}^{n+1}=\dot{Y}^{n}+\Delta t \ddot{Y}^{n}, \\
& Y^{n+1}=Y^{n}+\Delta t \dot{Y}^{n}+(\Delta t)^{2} \ddot{Y}^{n},
\end{aligned}
$$

with $Y^{1}$ and $\dot{Y}^{1}$ being initially prescribed the position and velocity of the cylinder, otherwise,

$$
\begin{aligned}
& \dot{Y}^{n+1}=\dot{Y}^{n}+\Delta t\left[(1-\gamma) \ddot{Y}^{n-1}+\gamma \ddot{Y}^{n}\right], \\
& Y^{n+1}=Y^{n}+\Delta t \dot{Y}^{n}+(\Delta t)^{2}\left[(0.5-\beta) \ddot{Y}^{n+1}+\beta \ddot{Y}^{n}\right],
\end{aligned}
$$

where $\gamma=0.5$ and $\beta=0.25, \ddot{Y}^{n}$ and $\ddot{Y}^{n-1}$ are obtained from Eq. (3) at $t=t_{n}=n * \Delta t$ and $t=t_{n-1}$ respectively, in which $F_{L}$ is obtained from solution of the Navier-Stokes equations. When $\dot{Y}^{n+1}$ and $Y^{n+1}$ 
are found, they will be used in the body surface boundary condition of Eqs. (1) and (2) to obtain $F_{L}$ at $t=t_{n+1}$.

In the cases considered in the present study, the cylinder is quite close to the wall boundary, and during the oscillation it may hit the wall boundary and bounce back in some cases. Here it is assumed that the bounce-back is fully elastic. The velocity and the position of the cylinder after it is bounced back from the wall boundary is determined by

$$
\begin{aligned}
& v^{n+1}=-v^{n}, \\
& Y^{n+1}=2 Y_{b}-\left(Y^{n}+v^{n} \Delta t\right),
\end{aligned}
$$

where $v^{n+1}$ and $v^{n}$ are respectively the velocities of the cylinder at the first step after the cylinder hits the wall $\left(t_{n+1}\right)$ and the last step before the cylinder hits the wall $\left(t_{n}\right), Y^{n+1}$ and $Y^{n}$ are the corresponding positions of the cylinder and $Y_{b}$ is the position of the wall. The direction of the velocity $v^{n}$ at $t_{n}$ is towards the wall and the direction of the velocity $v^{n+1}$ is away from the wall, which is reflected in Eq. (8). The bounce back process is assumed to complete within one time step. In order to avoid mesh failure in the simulation, the cylinder is forced to be bounced back when the cylinder continues to move towards the wall and the gap $e$ is smaller than $0.005 D$, which is similar to the treatment used by Zhao and Cheng[14].

\subsection{Problem setup}

The physical problem is modeled as flow past a near-wall circular cylinder of diameter $D$ in a rectangular computational domain (Figure 1). The size of the whole computational domain is $50 D$ in the flow direction and $10.5 D+e$ in the cross-flow direction. The origin of the coordinate system is located at the centre of the cylinder $\left(x_{c}=0, y_{c}=0\right)$. The inlet boundary is $20 D$ upstream, and the outlet boundary is located $30 D$ downstream. These boundaries are sufficiently far away and the effects of the boundary truncation on the cylinder can be neglected 21]. The top boundary is located at $10 \mathrm{D}$ above the centre of the cylinder.

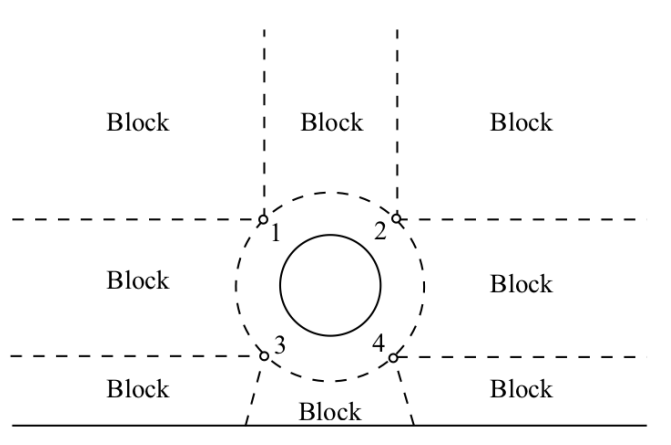

(a) Block sketch of computational mesh.

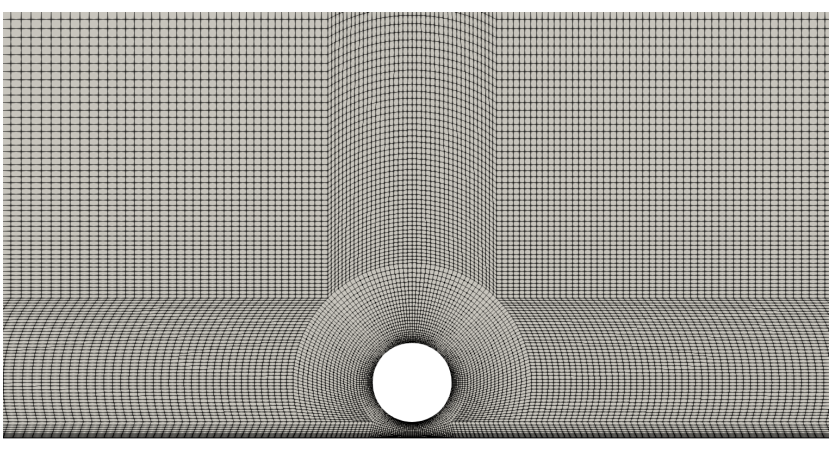

(b) Zoom-in mesh around the circular cylinder.

Figure 2: Mesh block sketch and zoom-in mesh. Dash lines denote block boundaries, hollow circles denote block nodes. The block nodes are equivalently moved based on the cylinder displacement in the remeshing process.

In the simulation, no-slip boundary condition is imposed on the wall and cylinder. At the flow inlet, a constant velocity is given for a uniform incoming flow. A slip boundary condition is applied to the upper boundary $(\partial u / \partial y=0$ and $\partial v / \partial y=0)$. At the flow outlet, the pressure is specified and a zero-gradient of the outflow flux is applied. For the cylinder motion, its position and velocity are obtained at each time step from Eqs. (6) to (7).

Computational mesh for the domain is generated using blockMesh dictionary. The computational domain is divided into multiple blocks in the mesh generation process (see Fig. 2a). A body-fitted O-mesh is adopted near the cylinder. A sketch of the zoom-in mesh near the cylinder for $e / D=0.2$ at the initial stage is illustrated in Fig. (2b). To ensure the numerical accuracy, the size of the element attached to the cylinder 
or to the seabed is set to meet the condition $\eta^{+}=u_{\tau} \eta / \nu<1$, where $u_{\tau}=\sqrt{\tau_{w} / \rho}$ with $\tau_{w}=\mu \frac{\partial u}{\partial \eta}$ denoting the shear stress on the corresponding boundary. Such a condition of $\eta^{+}$guarantees accurate prediction of the shear stress near the wall.

During the computation, the nodal points of the mesh are moved according to the updated cylinder position. It is common that the displacement is obtained by solving differential equation $\nabla \cdot\left(\lambda \nabla S_{i}\right)=0$, where $S_{i}$ denotes the displacement of the node points and $\lambda$ denotes the diffusivity model. In the present study, the mesh in the gap is very fine, and the displacement $\left(S_{i}\right)$ obtained by solving the above differential equation may sometimes exceed the element size, which may cause a mesh regeneration process to crash. To improve the mesh quality in the case of small gap, a remeshing method is developed by the blockmesh module of the OpenFOAM codes instead of calculating the displacement of each node. When advancing to a new time step in the computation, the cylinder position is updated by Eq. (7). Meanwhile the block nodes (hollow circles in Fig. 2a) are equivalently moved based on the cylinder displacement. With the block nodes moved, the coordinates of each node are recomputed using blockmesh codes, without changing the connectivity of the mesh. Assuming the displacement of each node is small during the remeshing, the flow variables at each cell in the new mesh is approximated by the corresponding values in the old mesh. Eqs. (1) and (2) are then solved in the new mesh for the flow variables at the next time step.

\section{Results and discussion}

The present study focuses on motions of a spring-mounted circular cylinder close to a plane boundary at small gap ratios $(e / D=0.5,0.4,0.3,0.2$ and 0.1$)$ at $R e=200$. Eq. (3) may be nondimensionalized as

$$
m^{*} \ddot{Y}^{*}+\frac{4 \pi \zeta\left(m^{*}+1\right)}{U^{*}} \dot{Y}^{*}+\frac{4 \pi^{2}\left(m^{*}+1\right)}{U^{* 2}} Y^{*}=\frac{2 C_{L}}{\pi},
$$
with $\rho_{f}$ being the fluid density, $\zeta=c /\left(2 \sqrt{k\left(m+m_{a}\right)}\right)$ is structural damping ratio and $U^{*}=U /\left(f_{n} D\right)$ is reduced velocity with $f_{n}=\frac{1}{2 \pi} \sqrt{k /\left(m+m_{a}\right)}$ being the natural frequency, $C_{L}=F_{L} /\left(0.5 \rho_{f} U^{2} D\right)$ is the lift coefficient. To get some features of motion at relatively larger amplitude vibration, the structural damping ratio is set as zero in the present study. Computations are made for different reduced velocity $U^{*}$ at a given fixed mass ratio and Reynolds number. The $m^{*}$ is set to 2.6, which is the same as that in Zhao 14 . Adjustment of the cylinder stiffness is then used to alter the reduced velocity.

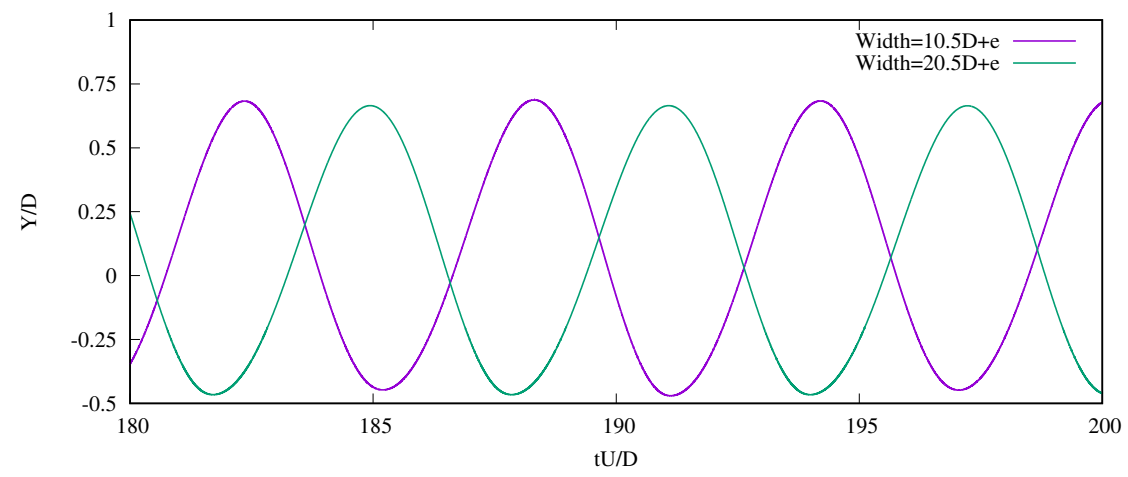

Figure 3: Time histories of the displacement of the cylinder at $U^{*}=6$ and $e / D=0.5$ with different domain width.

\subsection{Validations}

To check whether the blockage effect is of significance, computation is made respectively for width $10.5 D+e$ and $20.5 D+e$ at $U^{*}=6$ and $e / D=0.5$. Time histories of the displacement of the cylinder are 
shown in Figure 3. It can be seen that the results are virtually coincident. Figure 4 shows instantaneous vorticity contours when the cylinder is closest to the wall. The results from different width are very similar. These show that when the width is larger than or equal $10.5 D+e$, as in the present study, the blockage effect is insignificant.

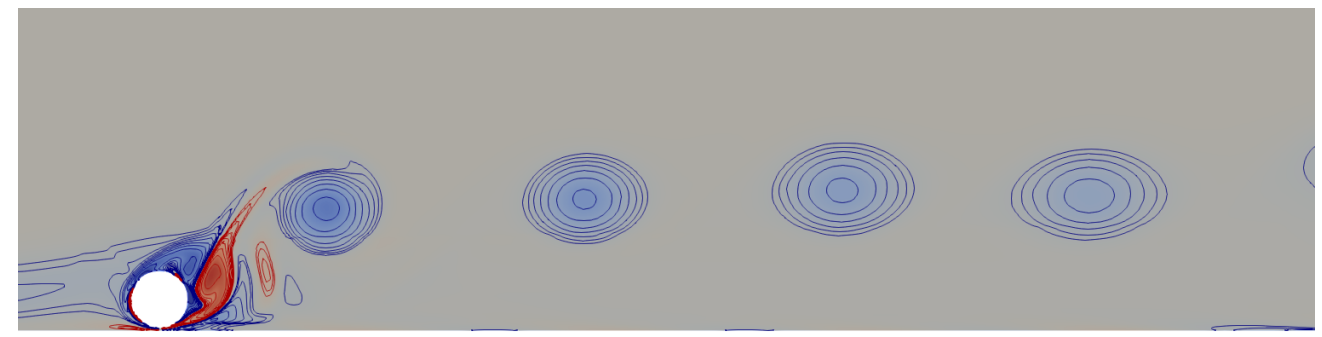

(a) Width $=10.5 D+e$

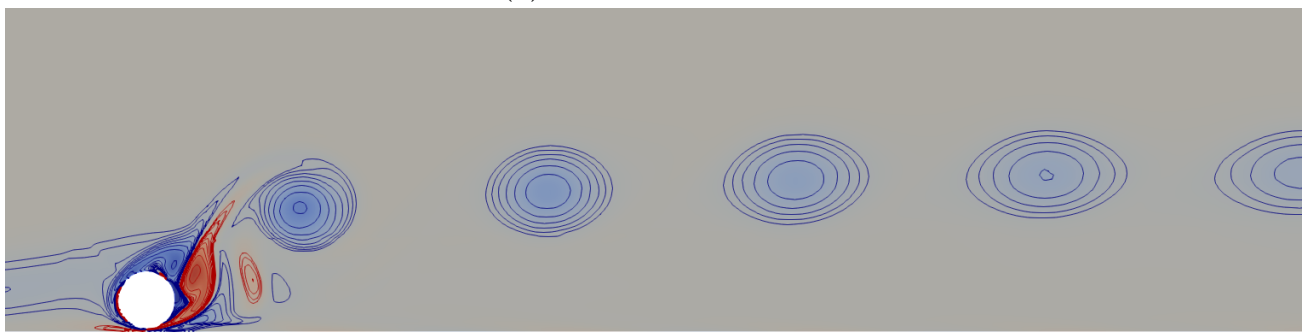

(b) Width $=20.5 D+e$

Figure 4: Instantaneous vorticity contours at $U^{*}=6$ and $e / D=0.5$ with different domain width (result is taken when the cylinder is closest to the wall).

To validate the present numerical method and computing codes, simulations of flows past a circular cylinder with $m^{*}=10$ at $e / D=0.5$ and $R e=100$, with two degrees of freedom, which are the same as those in Tham et al. [16], are performed. Studies have been conducted to ensure mesh convergence, as shown in Figure (5). The time step is determined by a Courant number limit of 0.1 and a maximum of $d t=0.001$. The figure shows the convergence and the finer mesh with 62313 cells is adopted for the further computations. The results including the maximum nondimensional transverse amplitude $A_{y}^{\text {max }} / D$, root-mean-square of longitudinal displacement $X_{r m s} / D, \operatorname{drag} C_{D, r m s}$ and lift $C_{L, r m s}$ coefficients, which are
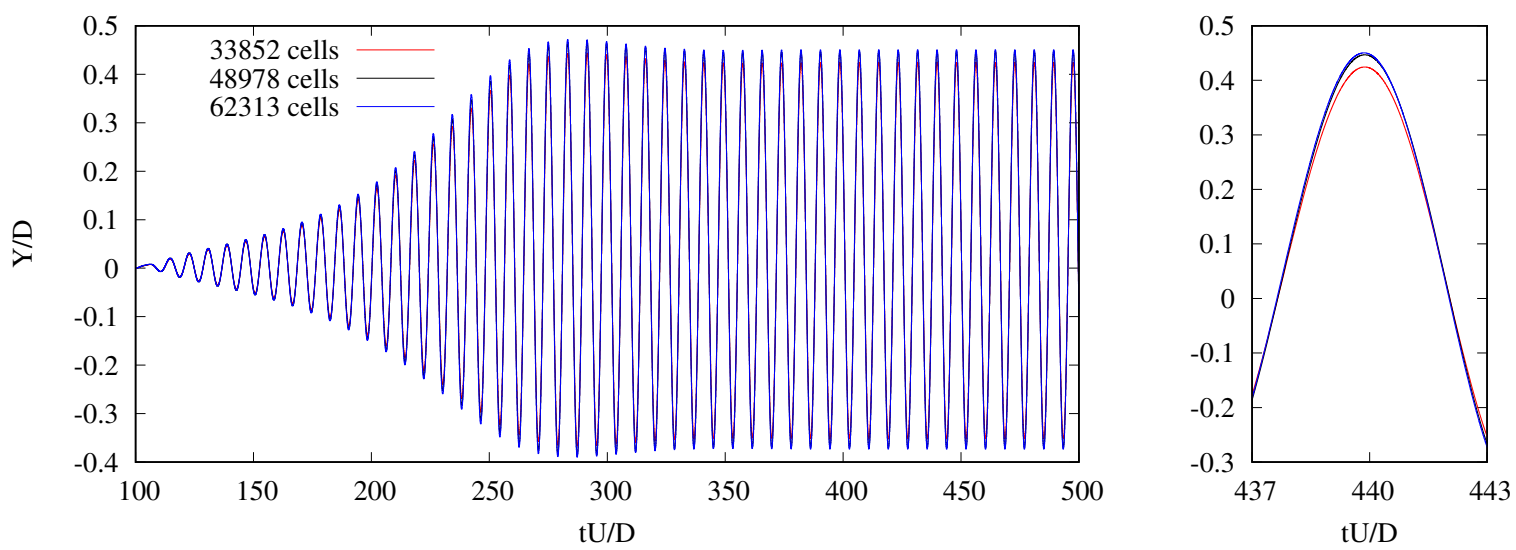

Figure 5: Cylinder displacement histories with different meshes at $m^{*}=10, \zeta=0, e / D=0.5, R e=100$ and $U^{*}=7.5$. 
calculated using at least 5 cycles of their corresponding time histories, are shown in Figure 6. Comparison is made with the results in [16] and the present results are generally in good agreement with those of Tham et al. except that around $U^{*}=9.5$ where the motion amplitude changes very rapidly.

(a) $A_{y}^{\max } / D$

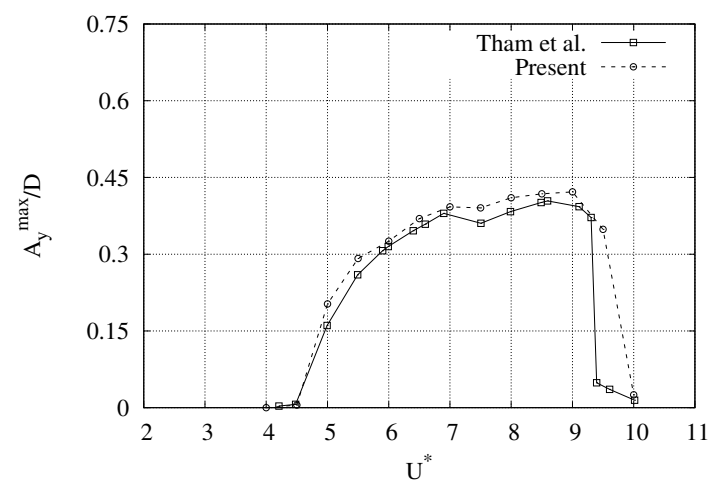

(c) $C_{D, r m s}$

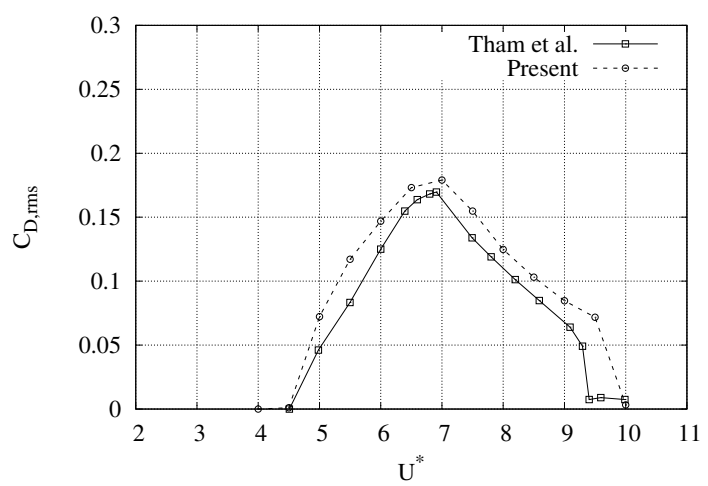

(b) $X_{r m s} / D$

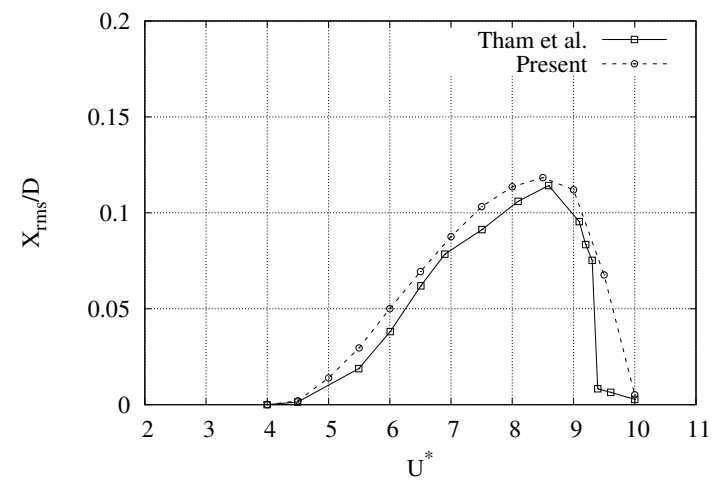

(d) $C_{L, r m s}$

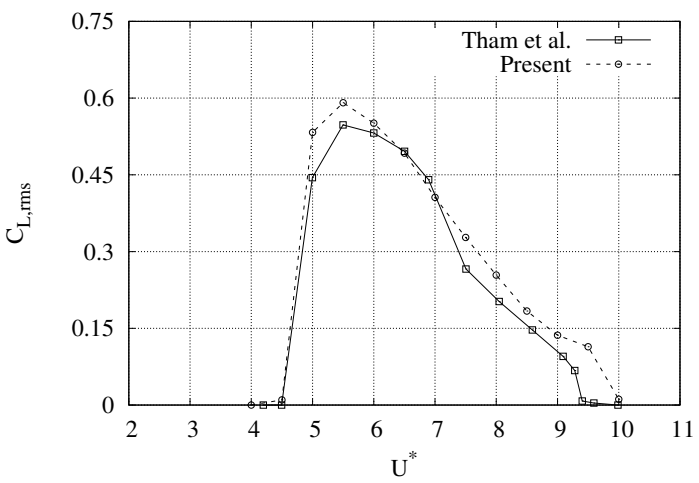

Figure 6: Comparison with Tham et al. [16] for a cylinder near the wall at $m^{*}=10, \zeta=0, e / D=0.5$ and $R e=100$.

\subsection{Displacement and hydrodynamic behavior}

Time histories of the transverse displacement and the lift coefficient of the cylinder $\left(m^{*}=2.6\right)$ and power spectrums of the transverse displacement and the lift coefficient at $e / D=0.5,0.3$ and 0.1 are shown in Figures 7, 8 and 9 respectively. The intermittency of the vibration response reported by $\mathrm{Li}$ et al.(2016) for a near-wall cylinder at relatively larger $e / D=0.9$ and by Leontini et al.[22, Prasanth and Mittal[21], Navrose and Mittal 23. for an isolated cylinder is not observed in the present study, and the vibration responses in all the cases are periodic. Figure 7 shows that the cylinder could be closest to the wall at $U^{*}=6$ but it does not collide with the wall at $e / D=0.5$. The frequency components of the lift and cylinder displacement, which are obtained by performing Fourier series expansions for $Y(t) / D$ and $C_{L}(t)$, are shown in the right column. It can be seen from Fig 7 that at $U^{*}=4, Y(t)$ and $C_{L}(t)$ are almost in phase and they both have one dominant frequency around $f_{n}^{*}$ in the energy level. As $U^{*}$ increases to 5 and 6 , while $Y(t)$ and $C_{L}(t)$ mainly remain in phase, other components of $C_{L}(t)$ become more visible, whose frequencies are multiples of the lowest frequency. At $U^{*}=7$ and 8 , the dominant frequency of $C_{L}(t)$ does not coincide with that of the $Y(t)$, and becomes about twice that of $Y(t)$. From Eq. (3), while the frequency components of $Y(t)$ and $C_{L}(t)$ may correspond to each other, their dominant components do not always coincide with each other. 
(a) $U^{*}=4, f_{n}^{*}=0.25$
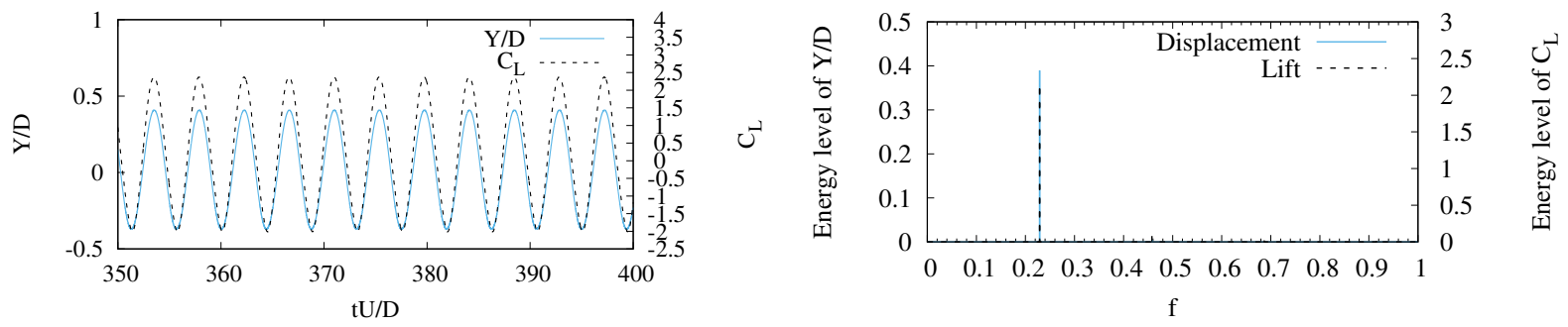

(b) $U^{*}=5, f_{n}^{*}=0.2$
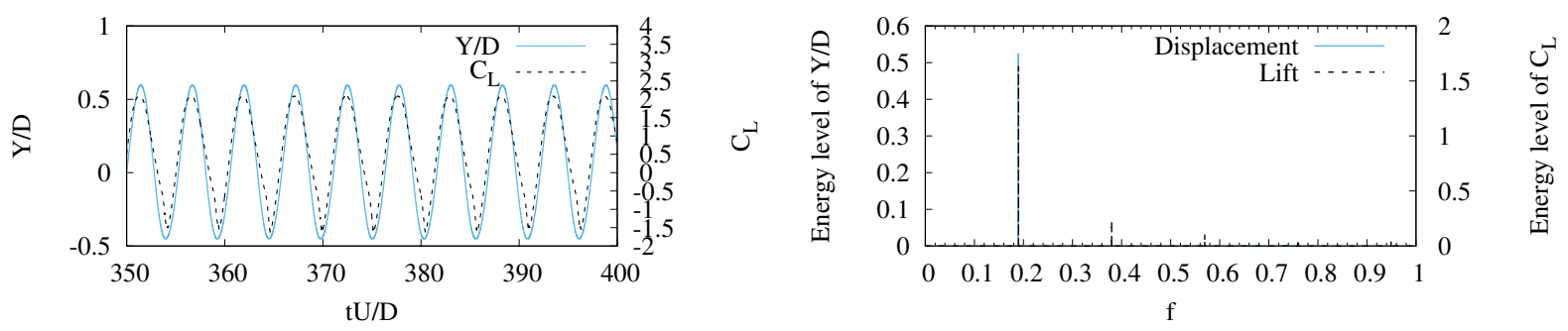

(c) $U^{*}=6, f_{n}^{*}=0.167$
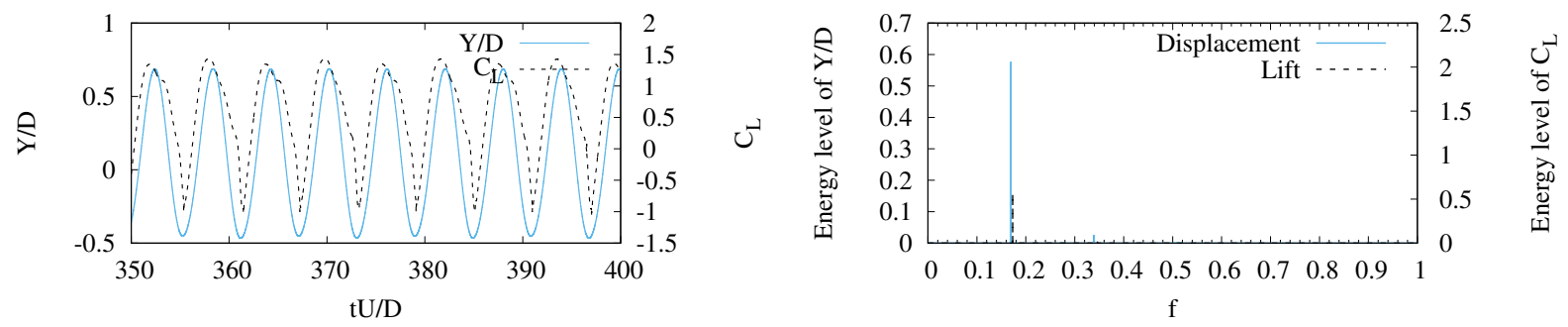

(d) $U^{*}=7, f_{n}^{*}=0.143$
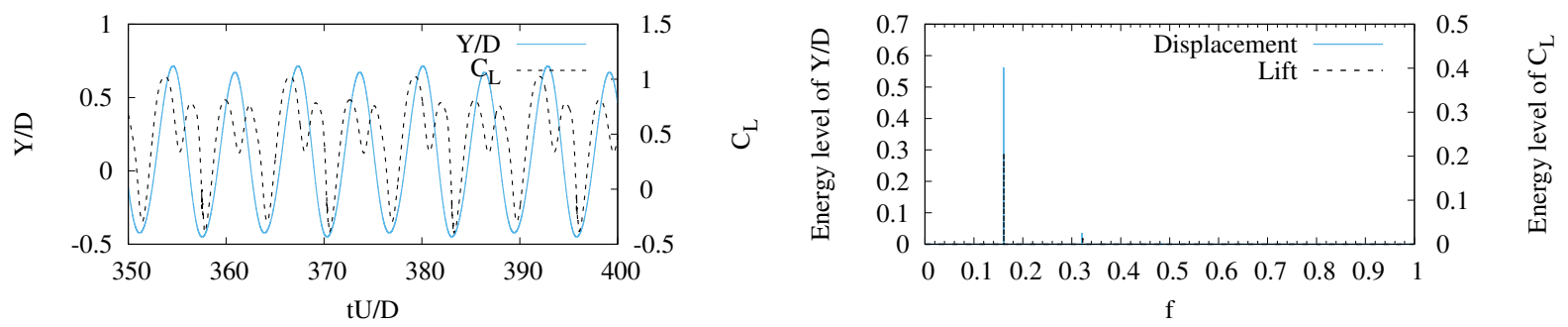

(e) $U^{*}=8, f_{n}^{*}=0.125$
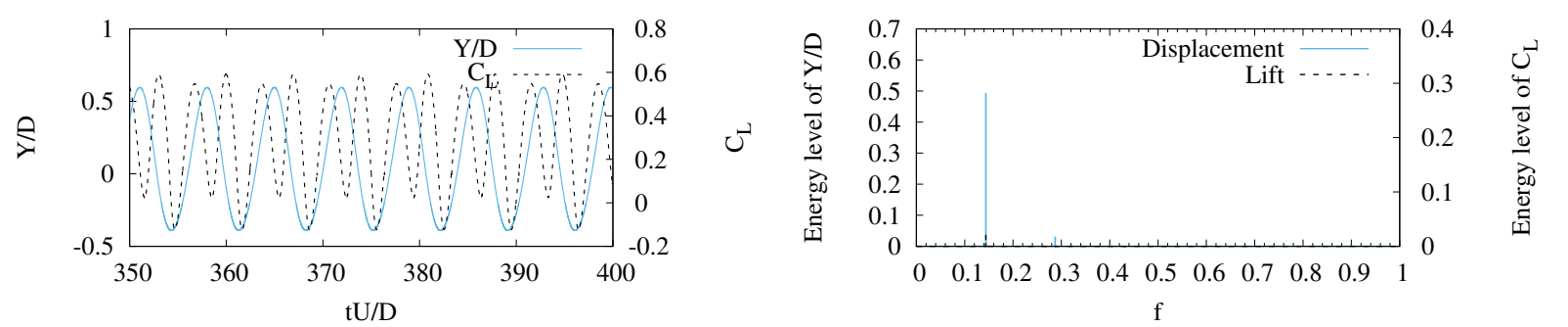

Figure 7: Time histories (left column), energy level (right column) of the cylinder displacement and lift force for $e / D=0.5 . f_{n}^{*}=1 / U^{*}$ is nondimensionalized natural frequency. 
(a) $U^{*}=5, f_{n}^{*}=0.2$
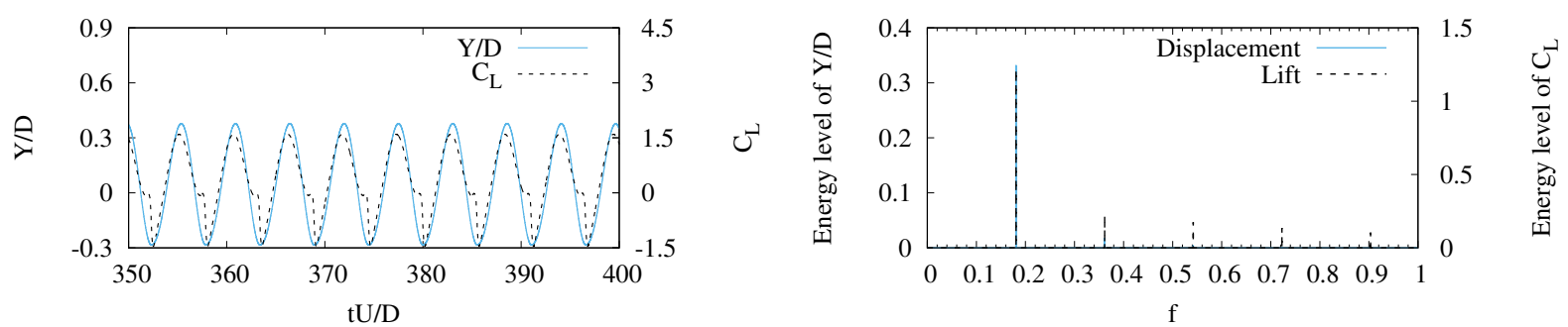

(b) $U^{*}=7, f_{n}^{*}=0.143$
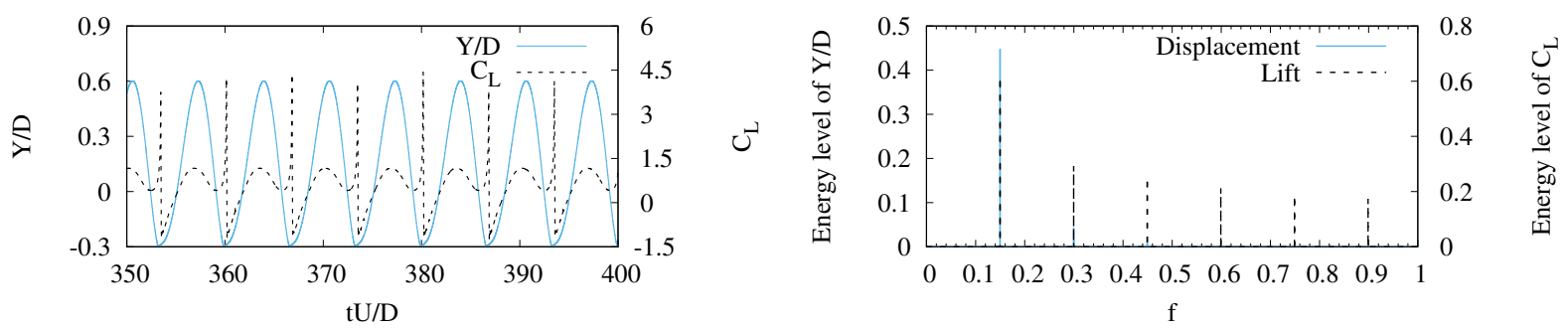

(c) $U^{*}=9, f_{n}^{*}=0.111$
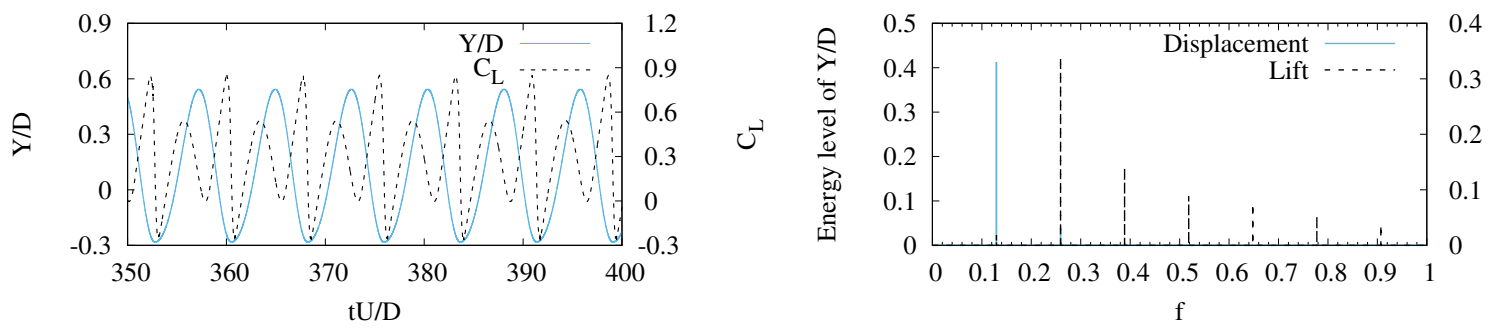

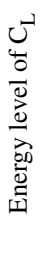

(d) $U^{*}=11, f_{n}^{*}=0.0909$
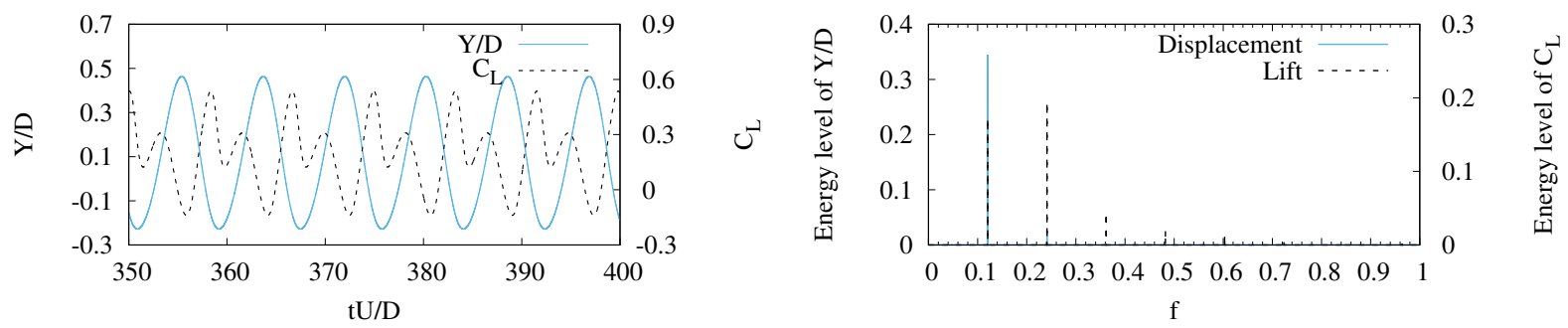

(e) $U^{*}=13, f_{n}^{*}=0.077$
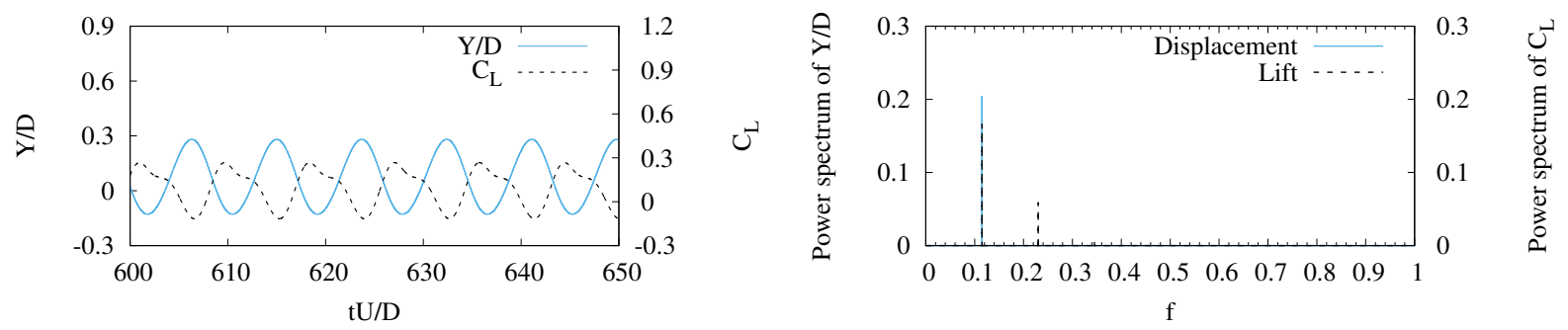

Figure 8: Time histories (left column), energy level (right column) of the cylinder displacement and lift force for $e / D=0.3 . f_{n}^{*}=1 / U^{*}$ is nondimensionalized natural frequency. 
(a) $U^{*}=6, f_{n}^{*}=0.1667$
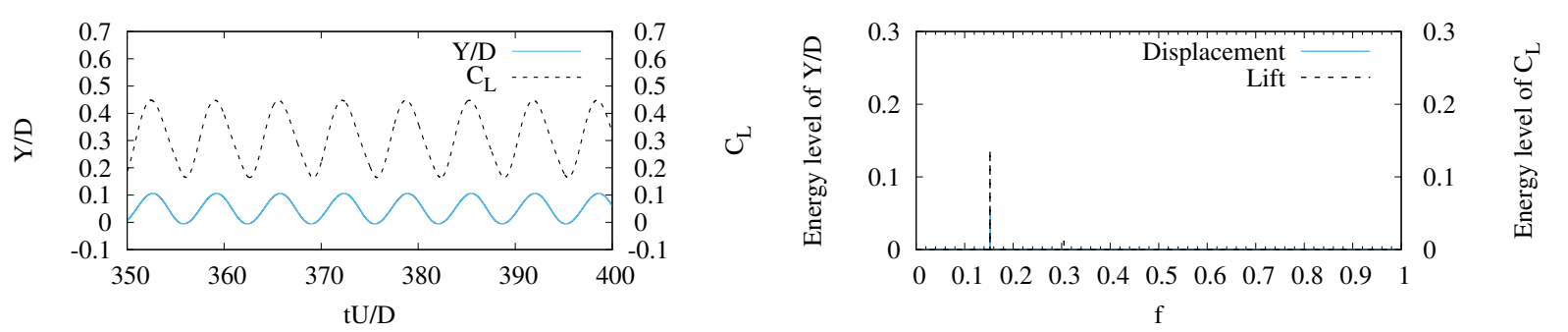

(b) $U^{*}=8, f_{n}^{*}=0.125$
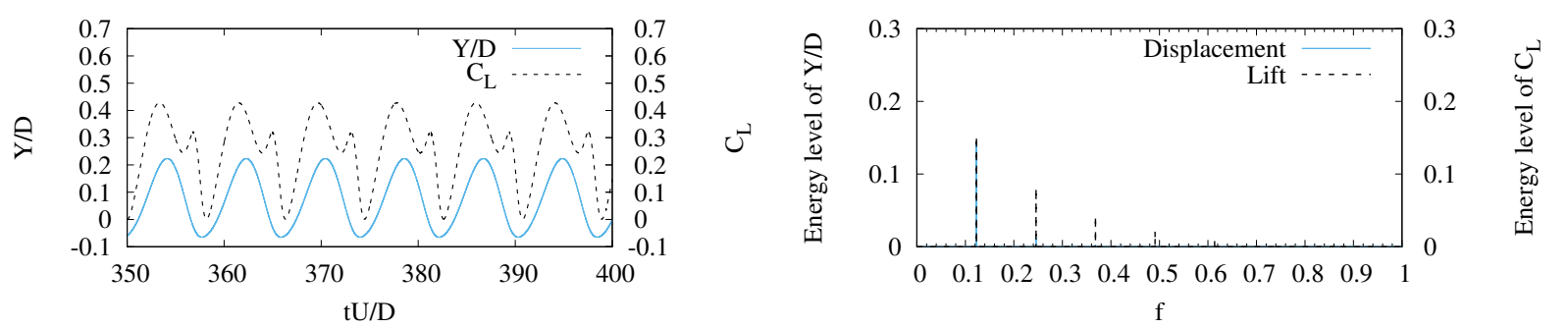

(c) $U^{*}=12, f_{n}^{*}=0.0833$
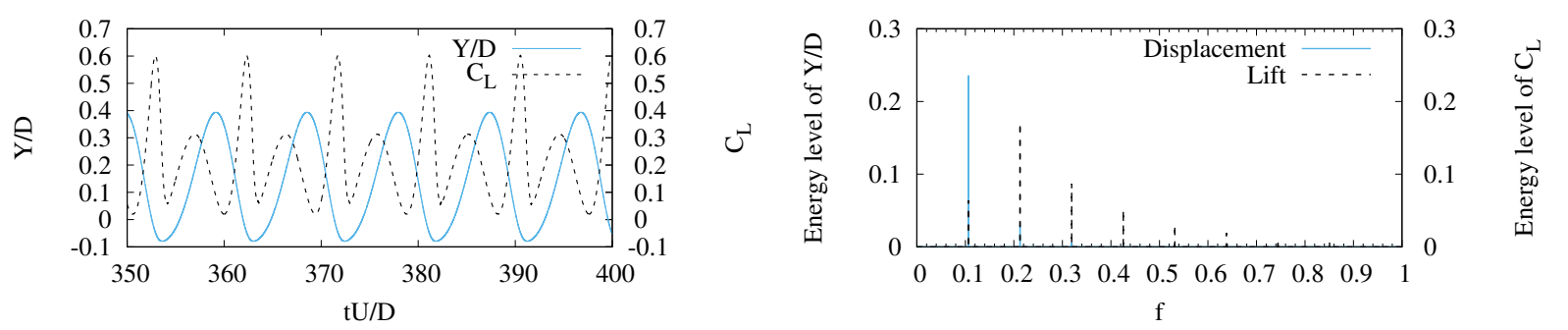

(d) $U^{*}=15, f_{n}^{*}=0.0667$
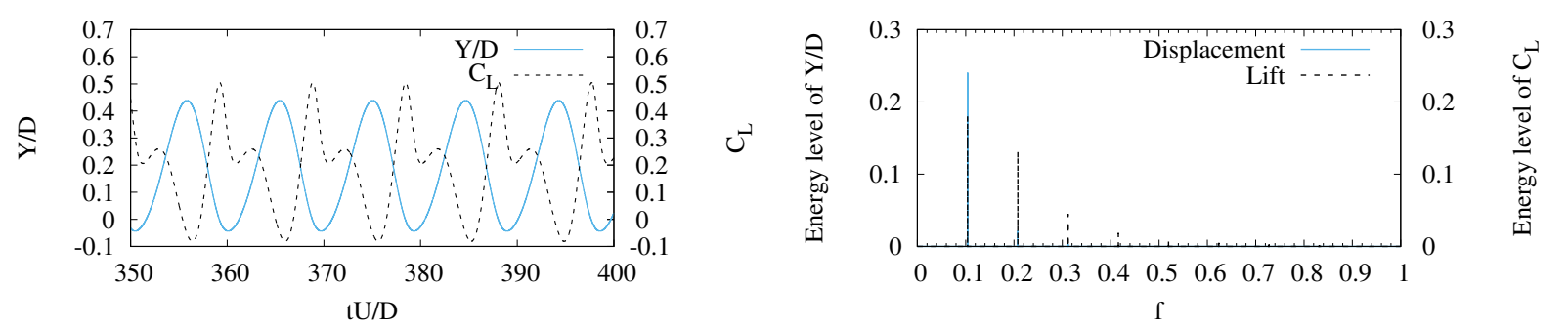

(e) $U^{*}=20, f_{n}^{*}=0.02$
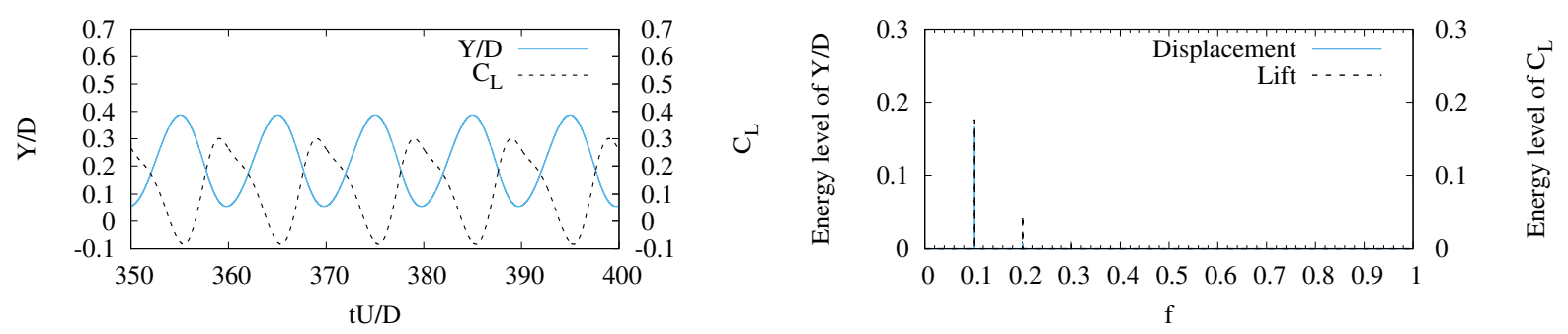

Figure 9: Time histories (left column), energy level (right column) of the cylinder displacement and lift force for $e / D=0.1 . f_{n}^{*}=1 / U^{*}$ is nondimensionalized natural frequency. 
Physically, it can be expected that $C_{L}(t)$ will be significantly affected by the displacement of the cylinder due to the wall effect, as well as by the velocity and acceleration. The coupling between $C_{L}(t)$ and $Y(t)$ will be nonlinear in general. The effects of displacement, velocity and acceleration on $C_{L}$ are similar to those due to the changes of stiffness, hydrodynamic damping and mass. For this reason, the natural frequency of an isolated cylinder is better predicted when the added mass is included in the mass or by the definition of $f_{n}$. However, it is important to realize that strictly speaking $f_{n}$ is an approximation. In particular, when the cylinder is near the wall, the equivalent stiffness will be affected by $e / D$, or $k$ will change, which is not reflected by the equation for $f_{n}$. Similarly, $m_{a}$ will also be affected by the wall. Therefore resonance may not happen exactly at $f_{n}$ and may happen at a frequency close to $f_{n}$ in many cases. Also when the resonance happens, the motion amplitude will depend on the equivalent hydrodynamic damping level from $C_{L}$ and the magnitude of the remaining $C_{L}$. In many cases, the motion amplitude may be biggest at resonance. However, it can be larger at different frequencies in other cases.

Further simulations are made for $e / D=0.3$ and the results are given in Fig 8 . At $U^{*}=5, Y(t)$ and $C_{L}(t)$ are mainly in phase. Their dominant frequencies coincide with each other and $f_{y} / f_{n} \approx 0.9035$. No collision of cylinder with the wall is observed. However at the trough of $Y(t)$, the bottom of the cylinder is very close to the wall. Just before that, $C_{L}(t)$ has a small increase over a short period of time before it continues to decrease. This leads to clearly visible components at other frequencies. The collision is observed in the case of $U^{*}=7$. The direction of the cylinder motion is reversed after the collision and correspondingly there is a sharp variation of $C_{L}(t) . C_{L}(t)$ then has multiple frequency components which are quite significant. However, $Y(t)$ is still dominated by the frequency of the first energy level peak with $f_{y} / f_{n} \approx 1.0486$. As $U^{*}$ increases, $Y(t)$ and $C_{L}(t)$ are gradually no longer in phase. Collision is not observed as $U^{*} \geq 9$. The dominant frequency of $C_{L}(t)$ becomes twice that of $Y(t)$ at $U^{*}=9$ and other components still stay significant since the trough of $Y(t)$ is very close to the wall. Meanwhile, $Y(t)$ and $C_{L}(t)$ become completely out phase. At $U^{*}=11$, the first two components of $C_{L}(t)$ are comparable, and the dominant

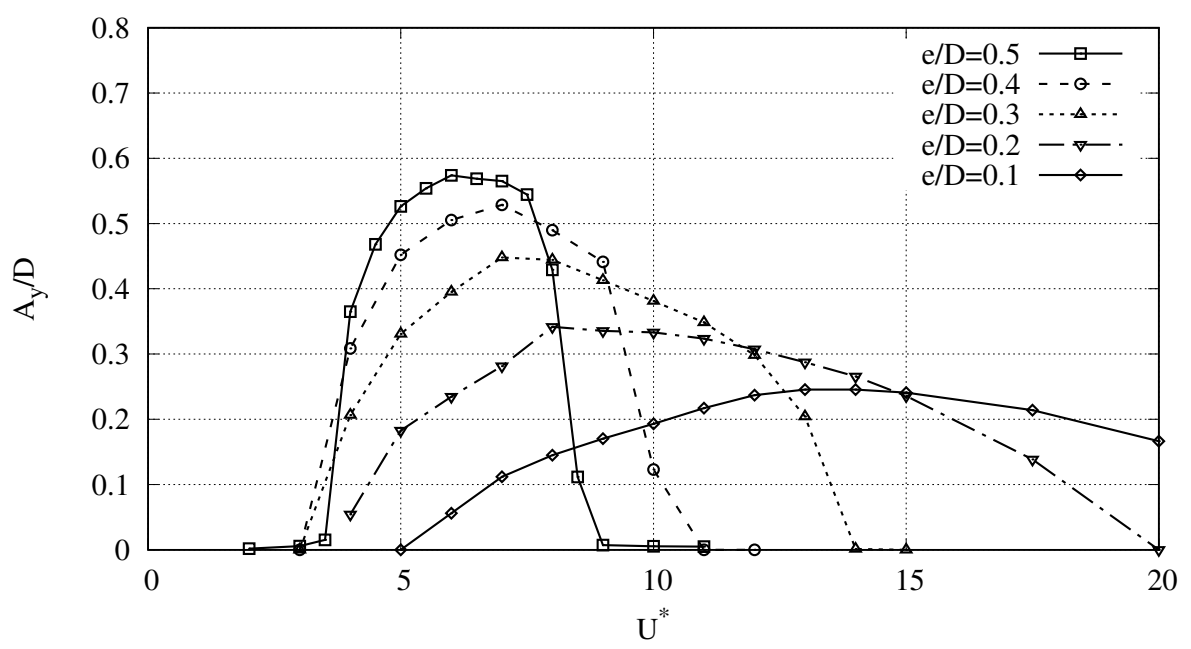

Figure 10: $A_{y}$ curves against reduced velocity for $e / D \leq 0.5$.

one is still twice that of $Y(t)$. As $U^{*}$ increases to 13 , the dominant frequency of $C_{L}(t)$ shifts back to be as the same as that of $Y(t)$ and other components become weak. $C_{L}(t)$ and $Y(t)$ become mainly anti-phase.

The results for $Y(t)$ and $C_{L}(t)$ at a smaller gap ratio $(e / D=0.1)$ are given in Figure 9. The figure shows that, in all the calculated cases with different $U^{*}$, the gap between the bottom of the cylinder does not reach the critical value at which the model for collision will be triggered. At $U^{*}=6$, there is a significant mean upward force in $C_{L}(t)$, which pushes the mean position of the cylinder upward. The dominant frequencies of $Y(t)$ and $C_{L}(t)$ coincide with each other. At $U^{*}=8$, the other components of $C_{L}(t)$ become significant, but at its largest peak, the frequency still coincides with the dominant frequency of $Y(t)$. This changes at 
$U^{*}=12$, where the dominant frequency of $C_{L}(t)$ is double that of the $Y(t)$. When $U^{*}=15$, it becomes similar to that at $U^{*}=8$. This continues at $U^{*}=20$. However, the components of $C_{L}(t)$ at other frequencies diminish, apart from the second one. $C_{L}(t)$ is mainly in-phase with $Y(t)$ at small $U^{*}$ (Figure 9a), and it becomes out phase as $U^{*}$ increases. At large $U^{*}$, the variation of $C_{L}(t)$ becomes mainly anti-phase with that of $Y(t)$ (Figure $9 \mathrm{e}$ ).

To further understand the free motion response of the cylinder, mean vibration amplitudes

$$
A_{y}=\frac{1}{2 N} \sum_{i=1}^{N}\left(y_{c, \text { max }}^{i}-y_{c, \min }^{i}\right)
$$

are calculated, where $i$ represents the $i$ th cycle of cylinder vibration, $N$ denotes the number of the vibration cycle, and $y_{c, \text { max }}^{i}$ and $y_{c, \text { min }}^{i}$ indicate the peak and trough in the period. The mean vibration amplitude is computed containing at least 10 cycles. Curves of $A_{y} / D$ against $U^{*}$ at different $e / D$ are plotted in Figure 10 It has been demonstrated that, in a laminar flow regime $(R e \approx) 100$ 24] or with low mass and structural damping 25, the cylinder response consists of two branches: initial and lower branches 25], between which there exists a hysteretic transition. In the current work, it can be observed that the vibration amplitude increases with $U^{*}$ first and then decreases after reaching a peak for each $e / D$ in $[0.1,0.5]$, and no hysteretic transition is observed. The figure shows that the largest vibration amplitude at each $e / D$ decreases with $e / D$. It is also noted that the reduced velocity associated with the largest amplitude increases as $e / D$ decreases, which results in different variations of the vibration amplitude with $e / D$ at different $U^{*}$. At low $U^{*}$, or $4<U^{*}<7.5$, the vibration amplitude decreases with $e / D$. With $U^{*}$ increasing, there appears a transition region, within which the vibration amplitude at large $e / D$ decreases and the one at small $e / D$ increases. At high $U^{*}$, the vibration amplitude at larger $e / D$ begins to diminish, while those at lower $e / D$

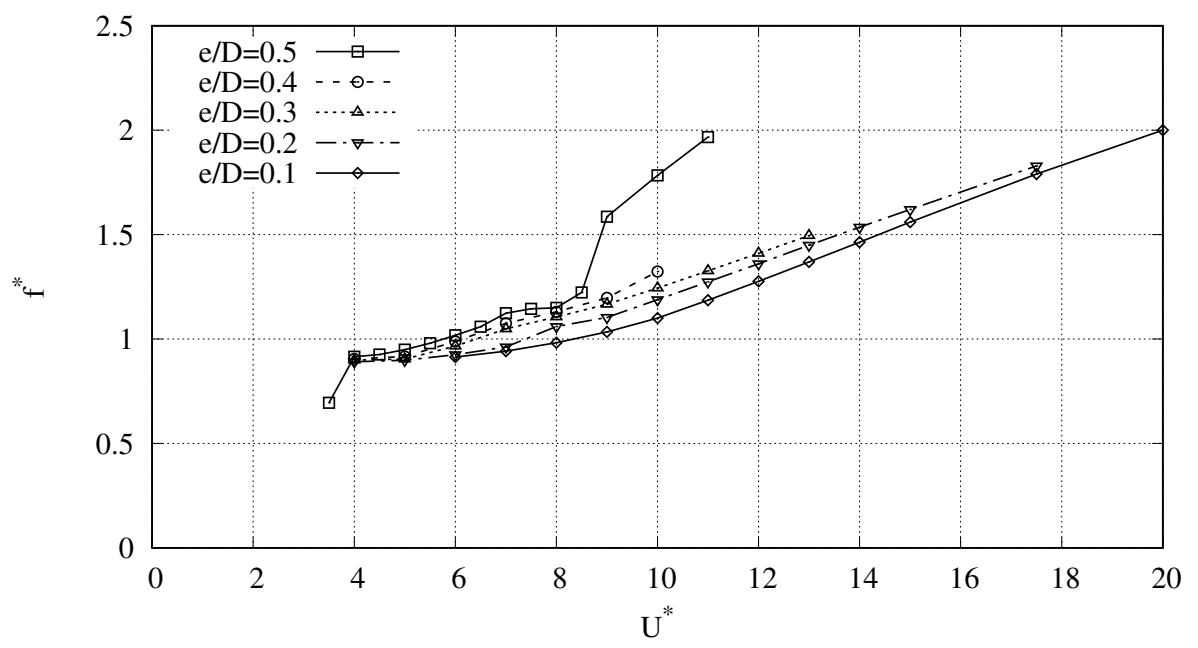

Figure 11: Variation of $f_{y} / f_{n}$ with reduced velocity.

are still significant. The amplitudes at lower $e / D$ also diminishes as $U^{*}$ further increases. However, the amplitude at $e / D=0.1$ remains significant even at $U^{*}=20$.

As discussed previously, when the cylinder is near a wall, the lifting force could be affected by the displacement of the cylinder. This effectively changes the stiffness of the cylinder, and therefore the natural frequency. This effect is not reflected in the equation of $f_{n}$ and therefore the equation is an approximation. Furthermore the potential flow added mass $m_{a}$ is also affected by the wall. Fig 11 shows the variation of $f^{*}=f_{y} / f_{n}$ against $U^{*}$ at different $e / D$. At smaller $U^{*}$ or the cylinder has relatively larger stiffness, $f_{y} / f_{n}$ in all the cases is close to 1 , from below 1 to above 1 as $U^{*}$ increases. Also as $U^{*}$ increases, $f_{y} / f_{n}$ increases generally at all $e / D$, and it increases more rapidly at larger $e / D$. 
When a cylinder is near a wall, the mean transverse force of the cylinder is due to three reasons: (1) even for a fixed cylinder, the vortex wake behind the body could be biased (2) the force due to body motion will have a non zero mean (3) the suction force due to the wall in the potential flow [26]. When the cylinder vibrates, it will move into a new mean position where the mean fluid force equals restoring force due to the mechanical stiffness. Figure 12 illustrates the variation of mean $Y(\bar{Y})$ of the cylinder with $U^{*}$ at different $e / D$. As small $U^{*}$ corresponds to a relatively large $k$, the cylinder is more like a fixed one, which is reflected by $Y \sim 0$, when $U^{*}<4$ in Figure 12 . As shown in Fig.10, the motion amplitude increases with $U^{*}$ initially. 275 Therefore, with the larger wake vortex triggered by the larger-amplitude motion, the mean lift due to the wake vortex also increases with $U^{*}$. When the motion amplitude in Fig 10 passes its peak and decreases with $U^{*}$, the wake vortex becomes weaker. $\bar{Y}$ also decreases as $U^{*}$ increases for $e / D=0.5,0.4$ and 0.3 . However, $\bar{Y}$ does not decrease with $U^{*}$ even at large $U^{*}$ for $e / D=0.2$ and 0.1 . This may be partly because at smaller initial gaps of $e / D=0.2$ and 0.1 , the reason (1) discussed above may still have large contribution. It can also be noticed that in the most cases, $\bar{Y}$ is positive or the force pushes the cylinder away from the wall. This indicates that the viscous force is larger than the potential suction force.

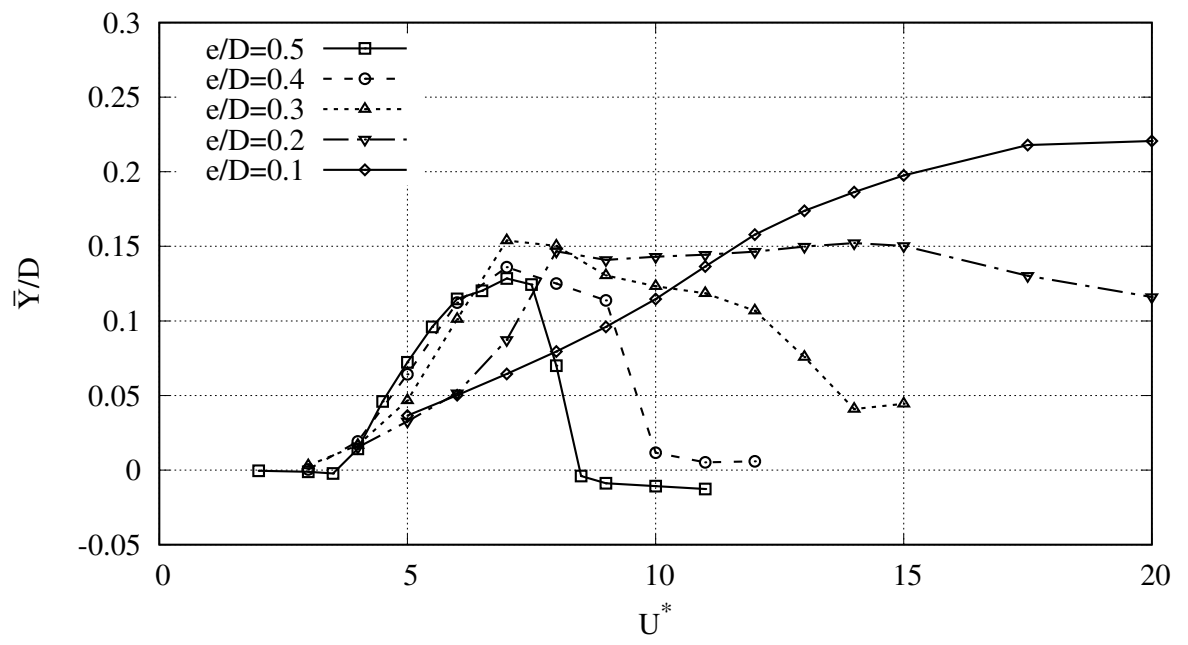

Figure 12: Variation of averaged displacement with reduced velocity.

\subsection{Vortex shedding}

The different branches in the response curve are associated with different vortex shedding modes at the wake of the vibrating cylinder ( $\mathrm{Li}$ et al. (2016) 17]). To investigate the evolution of vortex shedding during the vibration, four instantaneous vorticity contours, which are respectively at $\left(t_{1}\right) Y=\bar{Y}$ with the cylinder moving upwards, $\left(t_{2}\right) Y=Y_{\max },\left(t_{3}\right) Y=\bar{Y}$ with the cylinder moving downwards and $\left(t_{4}\right) Y=Y_{\min }$ in one motion cycle, are provided.

For $e / D=0.5,0.3$ and 0.1 respectively, Figures 13,14 and 15 provide evolutions of vorticity contours at the above four instants over a period for three different $U^{*} . Y(t)$ and $C_{L}(t)$ in the full period are shown in the first row, four instantaneous vorticity contours corresponding to $t_{1}, t_{2}, t_{3}, t_{4}$ are shown in the second to fifth rows. For the isolated cylinder, the " $2 S$ " vortex shedding mode indicating 2 single vortices and the " $2 \mathrm{P}$ " mode meaning 2 pairs of vortices shed in the wake per cycle were described in Williamson and Roshko (1988 27], 2004 28, 2008 29]) and Tang et al. [30. Govarhan and Williamson (2000) 31, Singh and Mittal (2005) 24], Prasanth and Mittal (2008) 21] found that the vortex shedding mode is primarily " 2 S", and " $2 \mathrm{P}$ " was observed when the cylinder underwent high-amplitude vibrations at low Reynolds numbers. With the wall effect, $\mathrm{Li}$ et al. 17 reported the existence of "1S" mode when the vibration amplitude was small and the "2S" mode appeared when the cylinder underwent large-amplitude vibration as $e / D=0.9$ at $R e=200$. For $e / D \leq 0.9$ at $R e=100$, only single vortex shedding modes were observed by Tham et al. (2015) [16]. 

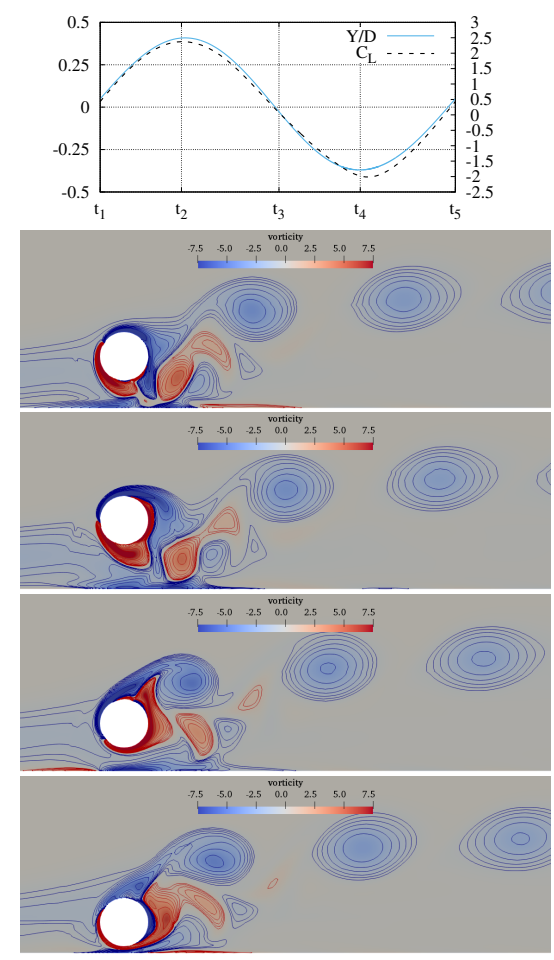

(a) $U^{*}=4$
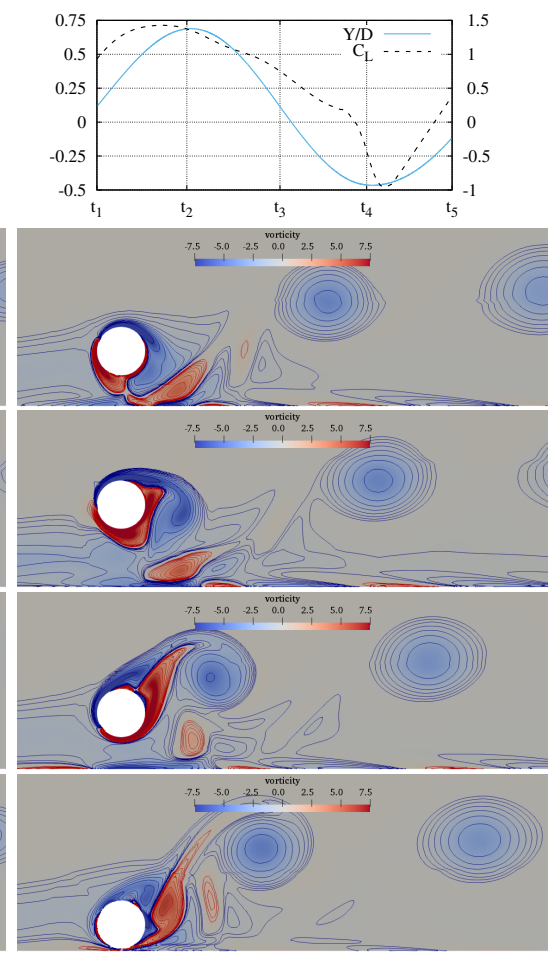

(b) $U^{*}=6$
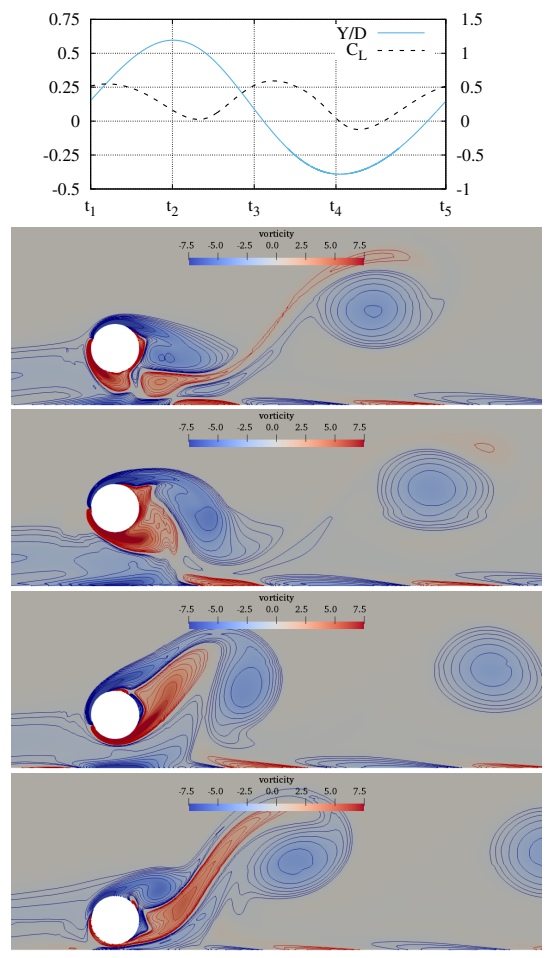

(c) $U^{*}=8$

Figure 13: Time histories of the cylinder displacement the lift (first row) and instantaneous vorticity contours at $t U / D=t_{1}, t_{2}, t_{3}$ and $t_{4}$ (from the second to fifth row) for $e / D=0.5$. Blue: negative (clockwise vortex), red: positive (anti-clockwise vortex).

In the present study, for $e / D=0.5$, periodic vortex shedding can be observed in the near wake, however the anti-clockwise vortices are suppressed and only one single vortex (" $1 \mathrm{~S}$ ) is shed downstream at $U^{*}=4,6$ and 8. Generally, due to the wall effect, the vortex street is deflected upwards. It can be observed that the distance between the two vortex cores increases with $U^{*}$, which indicates that the vortex shedding frequency in the wake increases when $U^{*}$ decreases. Moreover, at lower $U^{*}=4$ or higher frequency, the first clockwise (blue) vortex behind the cylinder is closer to the cylinder. As $U^{*}$ increases to 6 , the vortex moves away a bit from the cylinder. Due to the larger vibration amplitude, seen in Fig 10 the vortex street is biased upwards more when the cylinder moves towards the wall. When the cylinder is very close to the wall (at $\left.t_{4}\right)$, the anti-clockwise vortex in the near wake, shown in red, is more suppressed. At $U^{*}=8$, the first vortex in the wake is further away from the cylinder, and they are less biased as the vibration amplitude decreases.

When the initial position of the cylinder is closer to the wall at $e / D=0.3$, the clockwise vortex shed from the upper part of the cylinder remains strong. However, the anti-clockwise vortex from the lower part of the cylinder is relatively weaker, especially at lower reduced velocity, $U^{*}=4$. As $U^{*}$ increases the anti-clockwise vortex becomes stronger. The distance between the cores of clockwise vortexes increases and the vortex street behind the cylinder are biased upwards, which is similar to that in the case of $e / D=0.5$. At $U^{*}=7$, the bouncing-back model is triggered. The flow below the cylinder at t4 is therefore virtually blocked off and the anti-clockwise vortex from the wall is suppressed. At $U^{*}=13$, the collision model is not triggered any more, but the cylinder is quite near the wall at $t_{4}$. Both clockwise vortex from the cylinder and anti clockwise vortex from the wall are significant near the lower part of the cylinder.

When $e / D=0.1$, the vortex is less concentrated at its core and is relatively more evenly distributed. The vortex street is less biased upwards than those in the previous two cases. At $U^{*}=7$, the clockwise vortex from the wall is generally suppressed and anti-clockwise vortex from the lower part of the cylinder is 


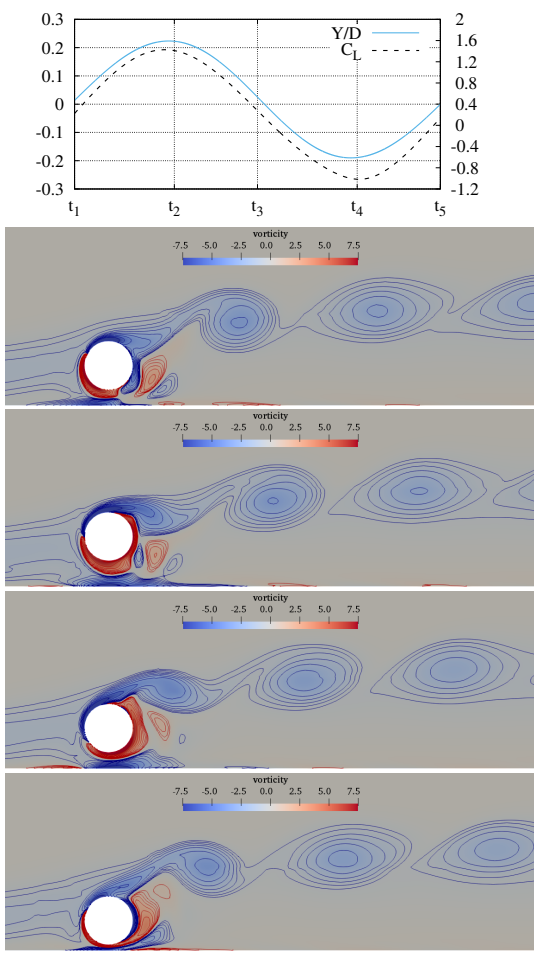

(a) $U^{*}=4$
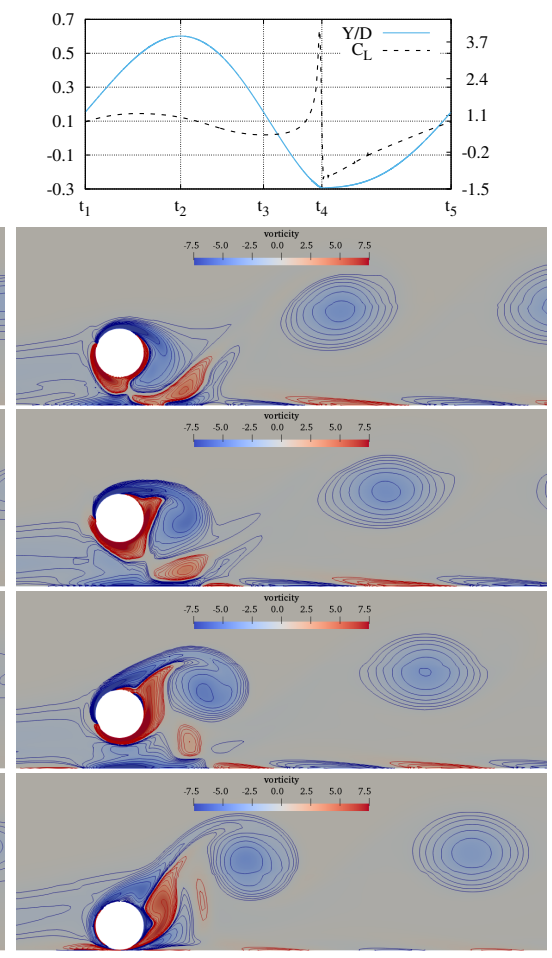

(b) $U^{*}=7$
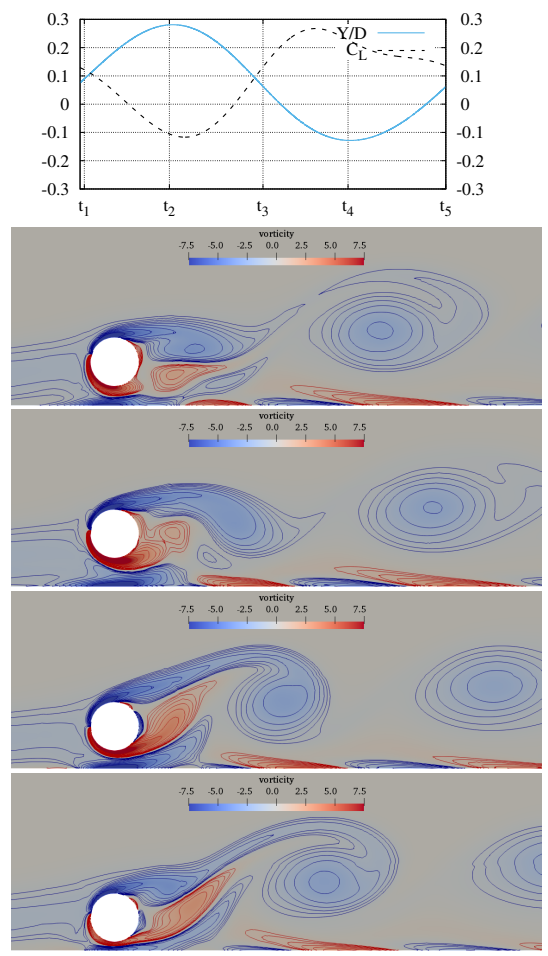

(c) $U^{*}=13$

Figure 14: Time histories of the cylinder displacement the lift (first row) and instantaneous vorticity contours at $t U / D=t_{1}, t_{2}, t_{3}$ and $t_{4}$ (from the second to fifth row) for $e / D=0.3$. Blue: negative (clockwise vortex), red: positive (anti-clockwise vortex).

relatively weak. The strengths of both of them increase at $U^{*}=14$. This remains the case at $U^{*}=20$.

\section{Conclusion}

Numerical studies on flow-induced transverse vibration of an elastically mounted rigid cylinder near a wall with small gap ratios at $R e=200$ have been performed, in which a remeshing technique is developed using OpenFOAM to prevent mesh over-distortion when the free motion cylinder is very close to the wall. In addition, a bounce-back model is adopted when the cylinder collides with the wall during the motion. The main conclusions from the present study can be summarized as follows:

1. In the simulations carried out, the lift force $C_{L}$ and displacement $Y$ show the periodic natures in time. $Y$ is very much dominated by a single frequency. $C_{L}$ may have a dominant frequency, but the components at other frequencies can also be significant.

2. At smaller reduced velocity $U^{*}$, the dominant frequency of $C_{L}$ coincides with that of $Y$. However, at large $U^{*}$, the dominant frequency of $C_{L}$ can be different from that of $Y$ and may become twice that of $Y$ in some cases.

3. The vibration amplitude increases with $U^{*}$ first and then decreases after reaching a peak, or there are only initial branch and lower branch in the cases simulated. When gap ratio reduces, the largest vibration amplitude decreases and the reduced velocity $U^{*}$ associated with the largest vibration amplitude increases.

4. The wall has significant effect on the equivalent stiffness of the cylinder. The frequency at which the largest amplitude occurs becomes larger than the natural frequency when gap ratio decreases. 


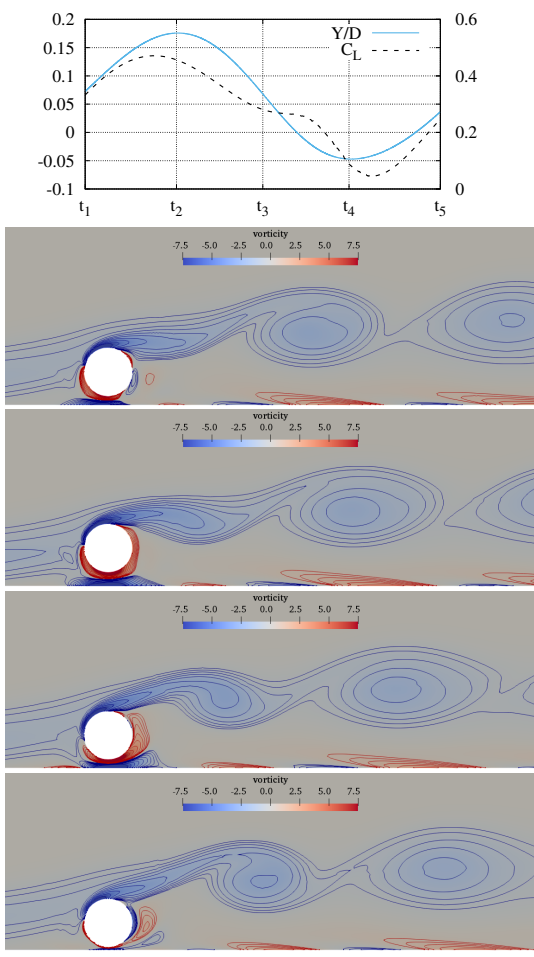

(a) $U^{*}=7$
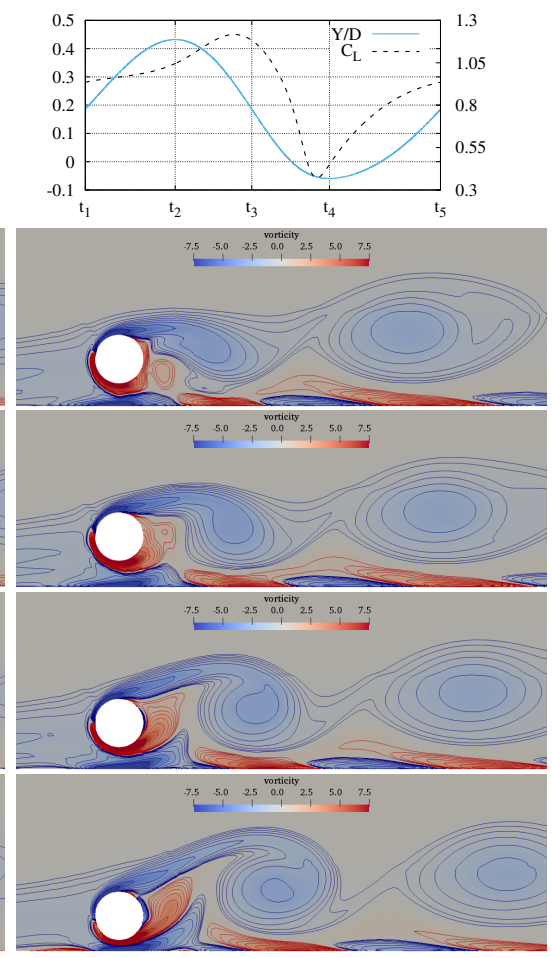

(b) $U^{*}=14$
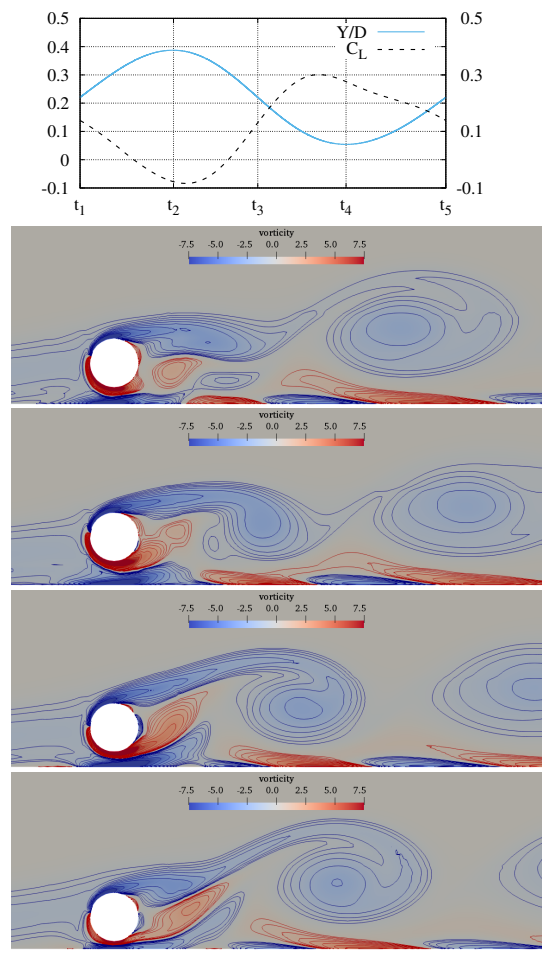

(c) $U^{*}=20$

Figure 15: Time histories of the cylinder displacement the lift (first row) and instantaneous vorticity contours at $t U / D=t_{1}, t_{2}, t_{3}$ and $t_{4}$ (from the second to fifth row) for $e / D=0.1$. Blue: negative (clockwise vortex), red: positive (anti-clockwise vortex).

5. The anti-clockwise vortices shed from the lower part of the cylinder are suppressed and only one single vortex ("1S) is shed downstream in the cases simulated. As $e / D$ decreases, the clockwise vortex shed from the upper part of the cylinder remains strong, the anti-clockwise vortex from the lower part of the cylinder gradually becomes weaker, and the vortex street is less biased upwards.

\section{Acknowledgments}

The authors wish to express our appreciation for the support provided by the Natural Science Foundation of Jiangsu Province (SBK2018040999), Natural Science Research of Jiangsu Higher Education Institutions of China (18KJB570001) and the National Natural Science Foundation of China (Grant No. 51879123).

\section{References}

[1] D. T. Tsahalis, The effect of seabottom proximity of the vortex-induced vibrations and fatigue life of offshore pipelines, Journal of Energy Resources Technology 105 (4) (1983) 464-468.

[2] B. M. Sumer, J. Fredse, Hydrodynamics around cylindrical structures, revised Edition, World scientific, 2006.

[3] P. W. Bearman, M. M. Zdravkovich, Flow around a circular cylinder near a plane boundary, Journal of Fluid Mechanics 89 (1) (1978) 33-47.

[4] M. M. Zdravkovich, Forces on a circular cylinder near a plane wall, Applied Ocean Research 7 (4) (1985) 197-201.

[5] C. Lei, L. Cheng, K. Kavanagh, Re-examination of the effect of a plane boundary on force and vortex shedding of a circular cylinder, Journal of Wind Engineering and Industrial Aerodynamics 80 (3) (1999) 263-286.

[6] X. K. Wang, S. K. Tan, Near-wake flow characteristics of a circular cylinder close to a wall, Journal of Fluids and Structures 24 (5) (2008) 605-627. 
[7] M. Zhao, L. Cheng, B. Teng, Numerical modeling of flow and hydrodynamic forces around a piggyback pipeline near the seabed, Journal of Waterway Port Coastal and Ocean Engineering 133 (4) (2007) 286-295.

[8] M. C. Ong, T. Utnes, L. Erik, D. Myrhaug, B. Pettersen, Near-bed flow mechanisms around a circular marine pipeline close to a flat seabed in the subcritical flow regime using a k- $\epsilon$ model, Journal of Offshore Mechanics and Arctic Engineering 134 (2) (2012) 021803.

[9] M. C. Ong, T. Utnes, L. E. Holmedal, D. Myrhaug, B. Pettersen, Numerical simulation of flow around a circular cylinder close to a flat seabed at high reynolds numbers using a k- $\epsilon$ model, Coastal Engineering 57 (10) (2010) 931-947.

[10] M. A. Prsic, M. C. Ong, B. Pettersen, D. Myrhaug, Large eddy simulations of flow around a circular cylinder close to a flat seabed, Marine Structures 46 (2016) 127-148.

[11] D. Tsahalis, W. Jones, Vortex-induced vibrations of a flexible cylinder near a plane boundary in steady flow, Proceedings of the Thirteenth Annual Offshore Technology Conferences (1981) 367-386.

[12] J. Fredse, B. M. Sumer, J. Andersen, E. A. Hansen, Transverse vibrations of a cylinder very close to a plane wall, Journal of Offshore Mechanics and Arctic Engineering 109 (1) (1987) 52.

[13] B. Yang, F. Gao, D. S. Jeng, Y. Wu, Experimental study of vortex-induced vibrations of a cylinder near a rigid plane boundary in steady flow, Acta Mechanica Sinica 25 (1) (2009) 51-63.

[14] M. Zhao, L. Cheng, Numerical simulation of two-degree-of-freedom vortex-induced vibration of a circular cylinder close to a plane boundary, Journal of Fluids and Structures 27 (7) (2011) 1097-1110.

[15] X. K. Wang, Z. Hao, S. K. Tan, Vortex-induced vibrations of a neutrally buoyant circular cylinder near a plane wall, Journal of Fluids and Structures 39 (5) (2013) 188-204.

[16] D. M. Y. Tham, P. S. Gurugubelli, L. Zhong, R. K. Jaiman, Freely vibrating circular cylinder in the vicinity of a stationary wall, Journal of Fluids and Structures 59 (2015) 103-128.

[17] Z. Li, W. Yao, K. Yang, R. K. Jaiman, B. C. Khoo, On the vortex-induced oscillations of a freely vibrating cylinder in the vicinity of a stationary plane wall, Journal of Fluids and Structures 65 (2016) 495-526.

[18] L. F. Chen, G. X. Wu, Boundary shear flow past a cylinder near a wall, Applied Ocean Research 92 (101923).

[19] R. Issa, Solution of the implicitly discretised fluid flow equations by operator-splitting, Journal of Computational Physics 62 (1) (1986) 40-65.

385 [20] G. X. Wu, Z. Z. Hu, A taylor series-based finite volume method for the navier-stokes equations, International Journal for Numerical Methods in Fluids 58 (2008) 1299-1325.

[21] T. K. Prasanth, S. Mittal, Vortex-induced vibrations of a circular cylinder at low reynolds numbers, Journal of Fluid Mechanics 954 (2008) 463-491.

[22] J. Leontini, M. Thompson, K. Hourigan, The beginning of branching behaviour of vortex-induced vibration during twodimensional flow, Journal of Fluids and Structures 22 (6) (2006) 857-864.

[23] Navrose, S. Mittal, Free vibrations of a cylinder: 3-d computations at re=1000, Journal of Fluids and Structures 41 (2013) 109-118.

[24] S. P. Singh, S. Mittal, Vortex-induced oscillations at low reynolds numbers: Hysteresis and vortex-shedding modes, Journal of Fluids and Structures 20 (8) (2005) 1085-1104.

25] A. Khalak, C. H. K. Williamson, Dynamics of a hydroelastic cylinder with very low mass and damping, Journal of Fluids and Structures 10 (5) (1996) 455-472.

[26] J. N. Newman, Analysis of small-aspect-ratio lifting surfaces in ground effect, Journal of Fluid Mechanics 117 (1982) 305-314.

[27] C. H. K. Williamson, A. Roshko, Vortex formation in the wake of an oscillating cylinder, Journal of Fluids and Structures 2 (4) (1988) 355-381.

28] C. H. K. Williamson, R. Govardhan, Vortex-induced vibrations, Annual Review of Fluid Mechanics 36 (1) (2004) $413-455$.

[29] C. H. K. Williamson, R. Govardhan, A brief review of recent results in vortex-induced vibrations, Journal of Wind Engineering and Industrial Aerodynamics 96 (6-7) (2008) 713-735.

[30] G. Tang, L. Lu, B. Teng, M. Liu, Numerical simulation of vortex-induced vibration with three-step finite element method and arbitrary lagrangian-eulerian formulation, Advances in Mechanical Engineering 5 (2013) 890423-890423.

[31] R. Govardhan, C. H. K. Williamson, Modes of vortex formation and frequency response of a freely vibrating cylinder, Journal of Fluid Mechanics 420 (2000) 85-130. 